$$
\text { تكامل إدارة القيمة وتحليل المخاطر بمشروعات التثييد والبناء. }
$$

- (INTEGRATED MODEL OF VALUE ENGINEERING AND RISK MANAGEMENT

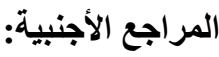
APPROACHES IN EMPOWERMENT PROJECTS (THE EXTERIOR DESIGN), BEHRANG ASKARI SABZKOHI, Department of Civil Engineering, Faculty of Engineering Islamic Azad University, Central Tehran Branch, Tehran, Iran, Proceedings of 17th IASTEM International Conference, Istanbul Turkey, 26th March 2016.

- Quantitative Risk Analysis and Integration of Value Engineering to Increase the Efficiency of Project Management Case Study: Sattar Khan Commercial Centre - Flour Parking Tehran, Parvaneh Shahsavand, Mediterranean Journal of Social Sciences MCSER Publishing, Rome-Italy, Vol 7 No 4 S2 August 2016.

-Tall Buildings Structural Systems and Aerodynamic Form Mehmet Halis Günel and Hüseyin Emre Ilgin.

-Project Management Istitute (Guide to the project Management Body of Knowledge)(Newtown square . Pennsylvania,USA 2017 6thEd).

- Al-Bahar, J and Crandall, K. (1990)."Systematic risk management approach for construction( projects”. Journal of Construction Engineering and Management, Vol.116, No.3

- H.M.Harris Jr, Creating value in engineering and construction frim , FMI corporation, New York , 2015. 
تكامل إدارة القيمة وتحليل المخاطر بمشروعات التشييد والبناء.

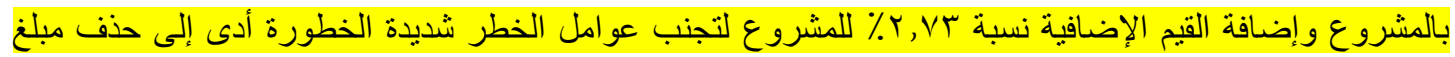

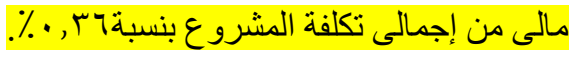

أما جودة المشروع تتحقق من الإدارة الرشيدة و التى يتم الدمج فيها بين الإدارتين لإز الة مواطن التكلفة غير الضـرورية

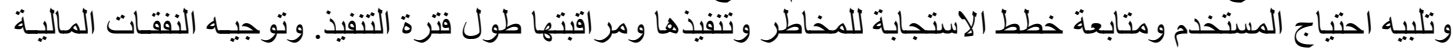

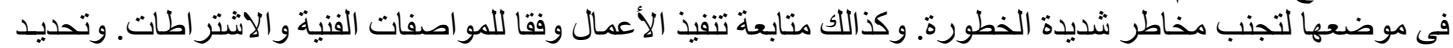

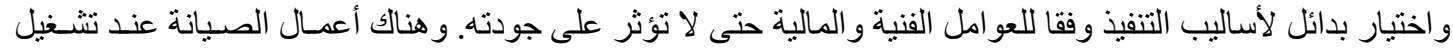

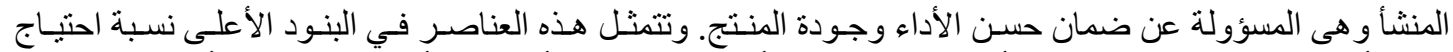

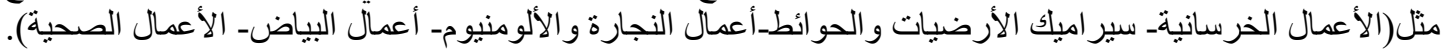

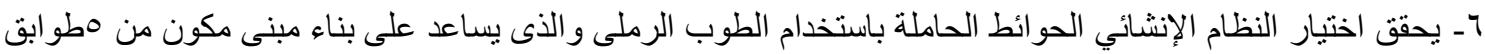

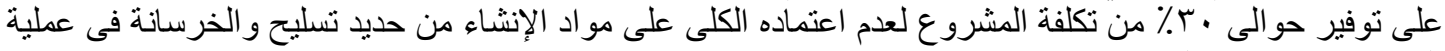
التشييد. و هو مناسب لمشرو هونات الإسكان.

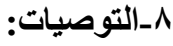

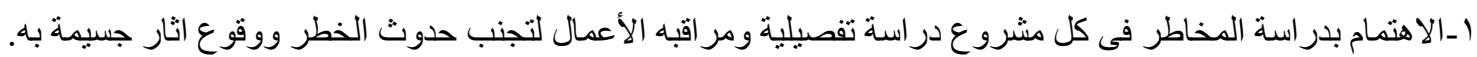

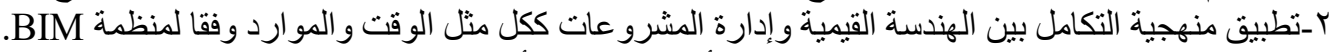

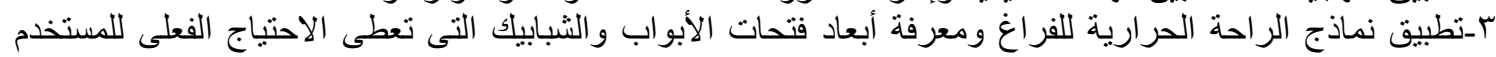

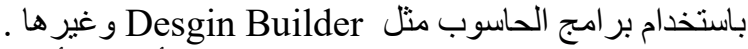

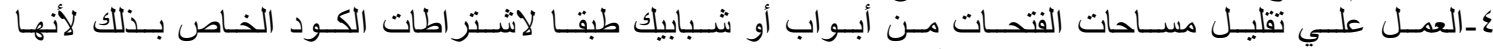

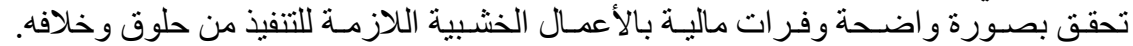

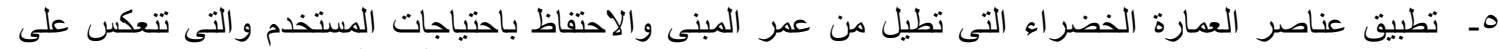

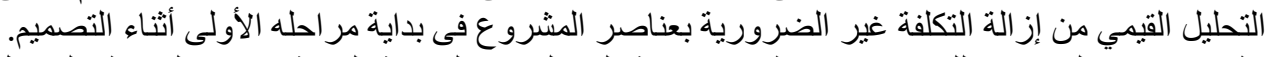

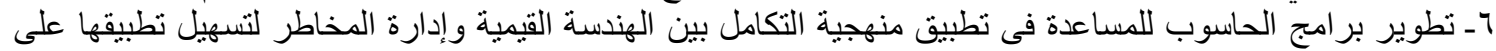

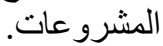

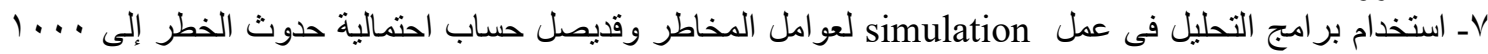

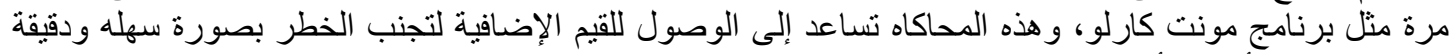
دون الوقوع فى أى خطأ حسابى.

9 المراجع العلمية:

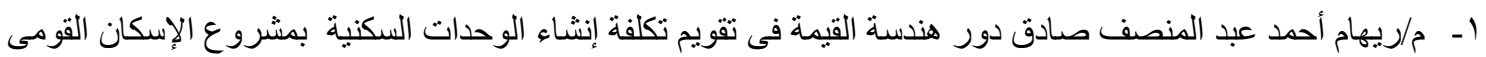

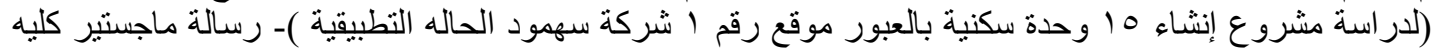

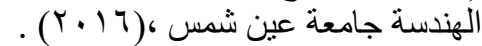
r- استخدام منهجية الهندسة القيمية في مشروع سكن الإيواء بهدف أمثلة الكلفة - رسالة ماجستير كلية الهندسة، جامعة

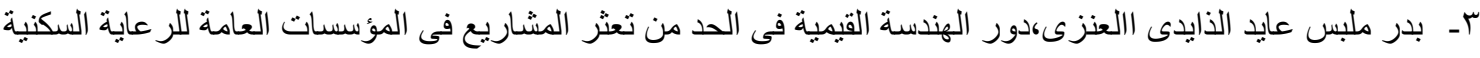

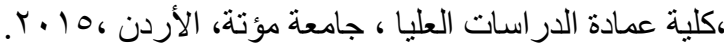

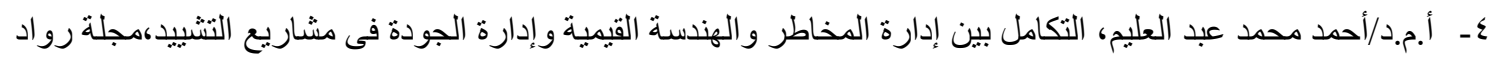

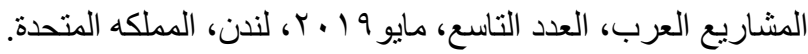

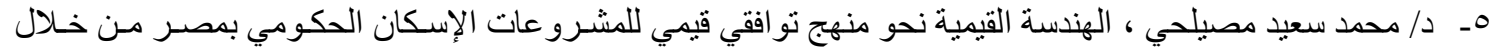

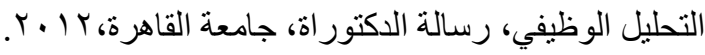

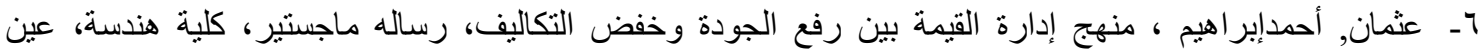

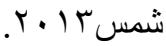

V- م/ ياسمين محمد مسعود،(تكامل منهجية الهندسة القيمية و إدارة عمليات الصيانة)، مجلة كلية الهندة جامعة الفيوم، r. 1 . ^ـ ـ المركز القومى لبحوث الإسكان والبناء، قسم العمارة والإسكان، دراسة توثيقية تقييم تجربة السكنى فى التجمعات

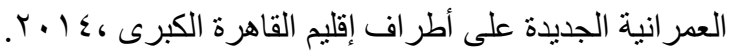


تكامل إدارة القيمة وتحليل المخاطر بمشروعات التثشييد والبناء.

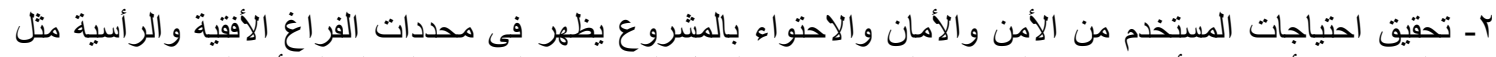

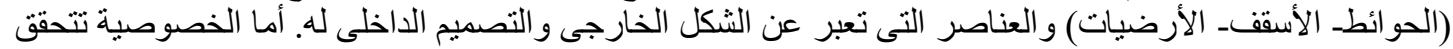

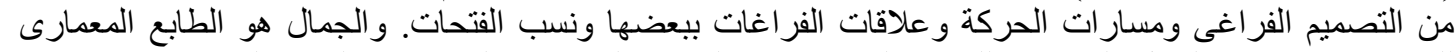

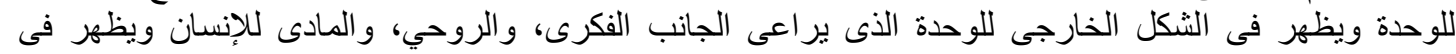

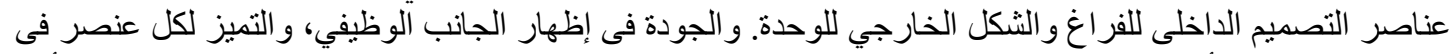
الوحدة. و المشكل الأساسى فى الاحتياجات الوظيفية تصميم الفراغ واغل علاقات الفر اغات الفات ببعضها و الحركة المطلوبة لتأدية

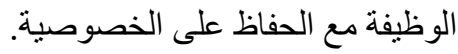

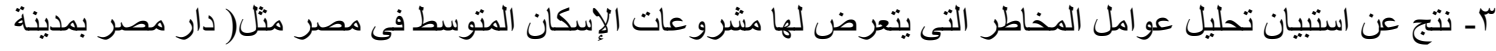

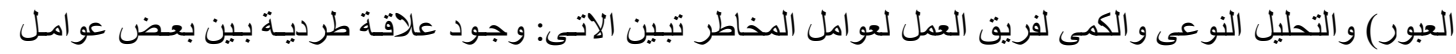

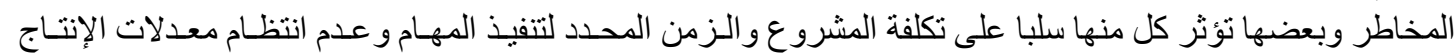

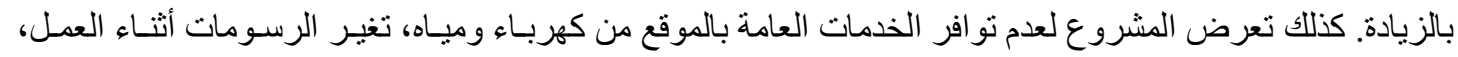

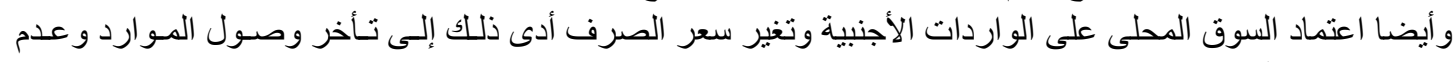

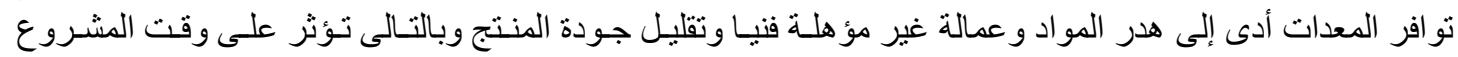
و انتظام الإنتاج.

ع - من خلال نو اتج استبيان التحليل الوظيفي للقيمة وتحليل المخاطر لشروع (دار مصر بمدينة العبور ): تم تحديد العناصـر

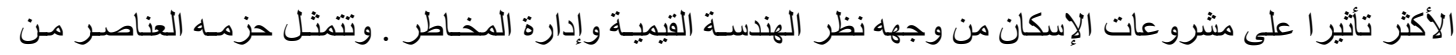

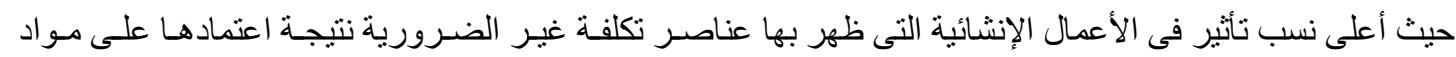

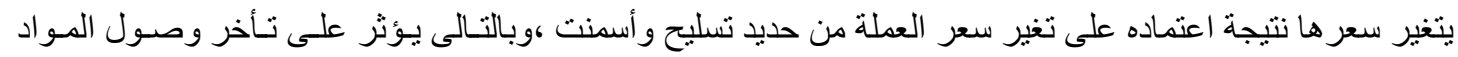
وتأخر مدة تنفيذ الأعمال وبالتالى يؤثر على التكلفة الكلية للمشروع.

و أيضا ظهرت فى الهيكل الإدارى نتيجة اضطر اب الدمج بين الإدارتين أثر ذلك على تـأخر الرسـومات وتنغير هـا أثتـاء

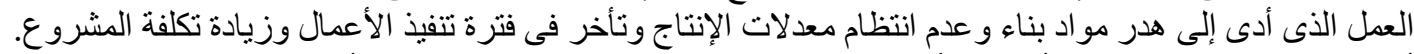

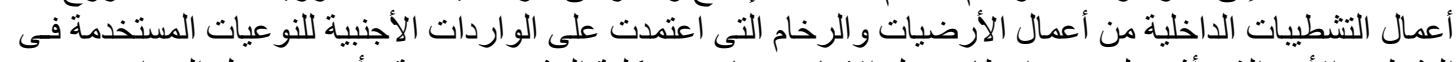

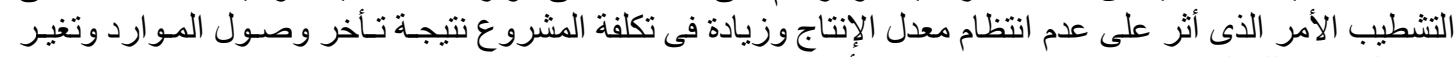

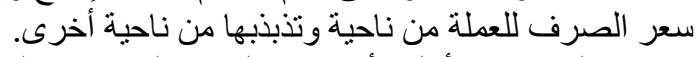

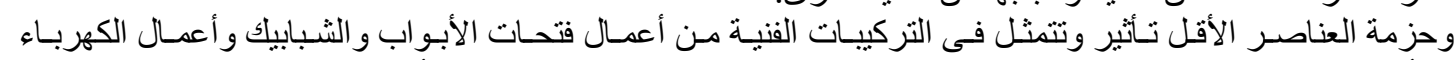

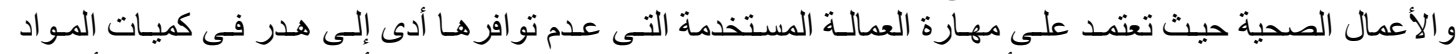

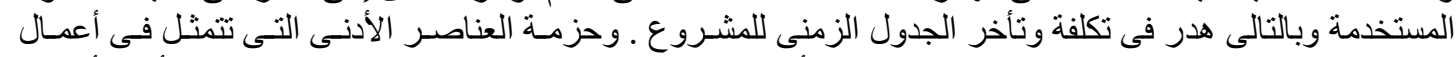

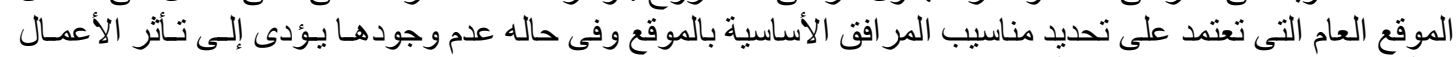
بالتأخير فى وقت إنجاء تعاز ها مثل الأعمال الصحية و الكهرباء.

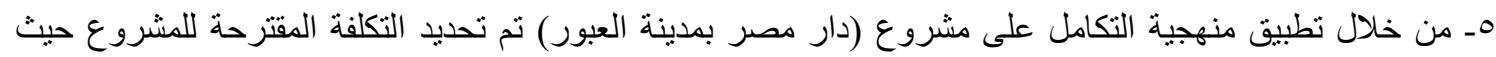

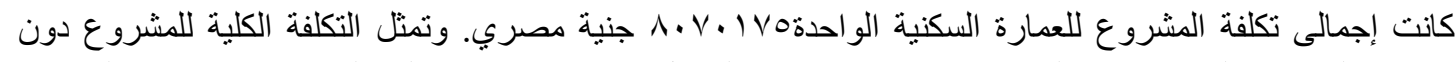

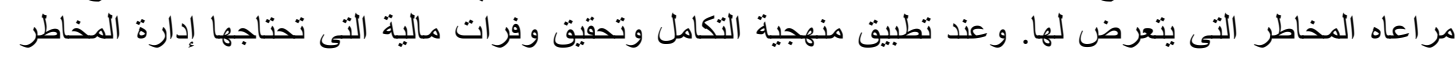

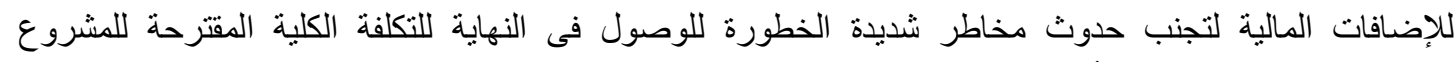

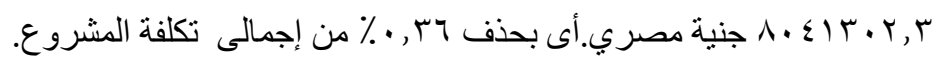

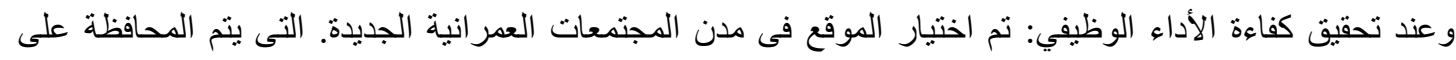

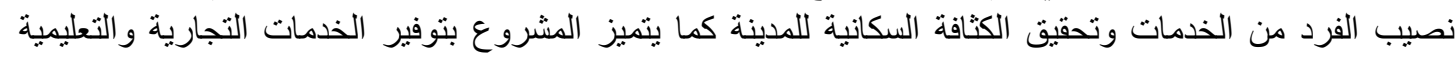

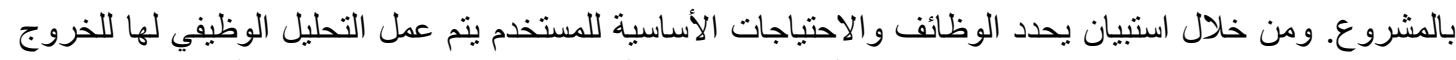

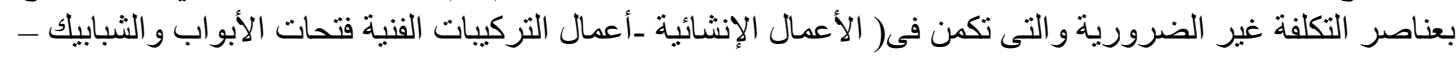

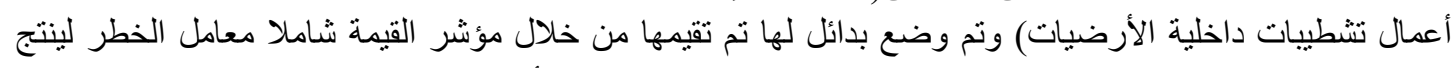

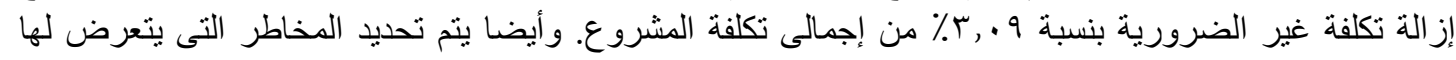

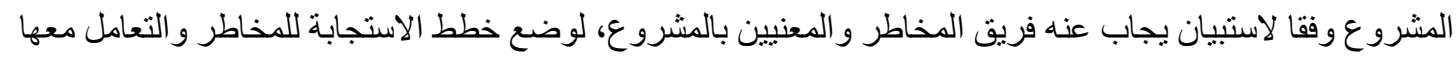

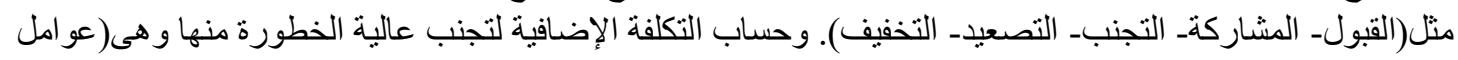

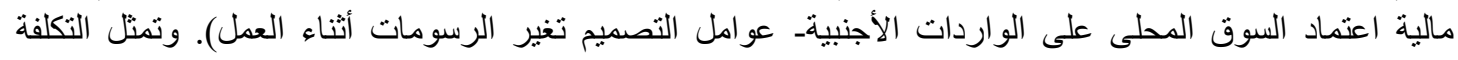

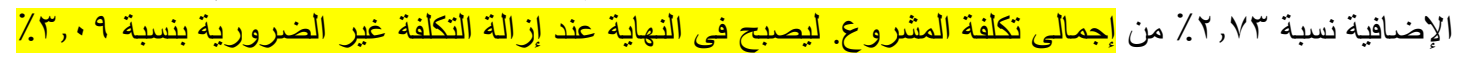


تكامل إدارة القيمة وتحليل المخاطر بمشروعات التشييد والبناء.

\begin{tabular}{|c|c|}
\hline التكلفة الإضافية & عامل الخطر \\
\hline 1970. & تغير الرسومات أثثاء العمل \\
\hline $1 r \cdot \lambda \cdot 9,94$ & اعتماد السوق المحلى على الواردات الأجنبية \\
\hline
\end{tabular}

ونستتتج من خلال التحليل وتطبيق منهجية التكامل بين الهندسة القيمية وإدارة المخاطر على مشروع دار مصر بمدينة

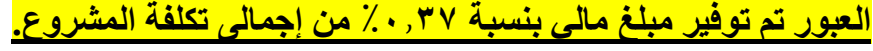

\begin{tabular}{|c|c|c|}
\hline المشروع بالكامل ( • ع عمارات ) & العمارة الواحدة & \\
\hline$\Lambda \cdot v \cdot I \vee 0$ & $\Lambda \cdot v \cdot 1 \vee 0$ & تكلفة المشروع الأساسية قبل تطبيق منهجية التكامل \\
\hline$r \leq q T r Y \leq .7$ & r $\{q r r Y . \leqslant \checkmark$ & 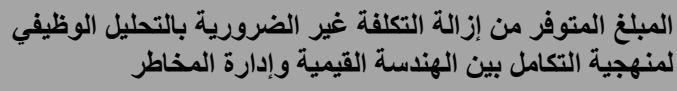 \\
\hline \multicolumn{2}{|l|}{$\%$ r.. 9} & النسبة المئوية للمبلغ المتوفر بتطبيق منهجية الهندسة القيمية \\
\hline$r r . \leqslant 0 q V . T$ & $r r . \leqslant 0 q . V q$ & 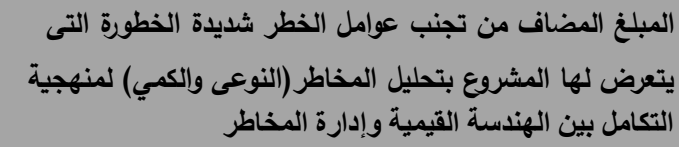 \\
\hline \multicolumn{2}{|l|}{$\%$ r.vr } & النسبة المئوية للمبلغ المضاف بالنسبة للمشروع \\
\hline YAAVYV & YAAVY.V & الإضافة وحذف المبالغ الناتجة من تطبيق منهجية التكامل بين \\
\hline \multicolumn{2}{|l|}{ 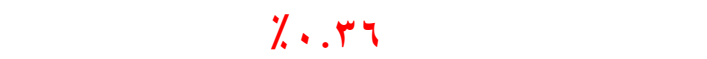 } & النسبة المئوية للمبلغ المتوفر النهائى للمشروع \\
\hline
\end{tabular}

: V Vتائج البحث

ا ـ من خلال العوامل المشتركة بين الهندسة القيمية وإدارة المخاطر التى يتعرض لها المشروع( التكلفةـ كفاءة الاداء

$$
\text { الوظيفي- الجودة) نستنتج الاتى: العنى }
$$

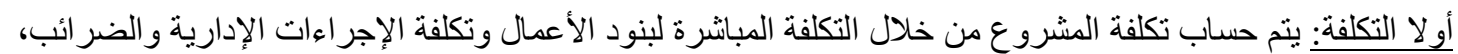

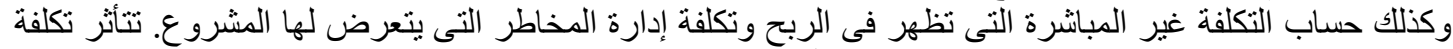

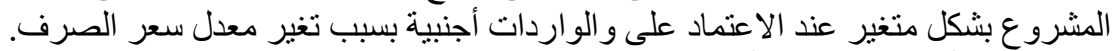

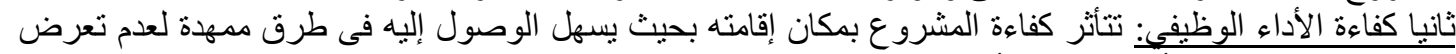

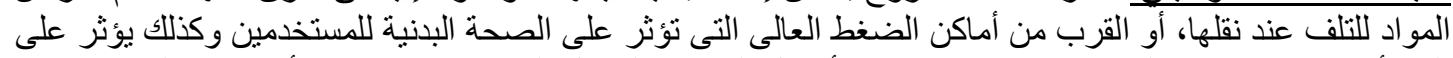

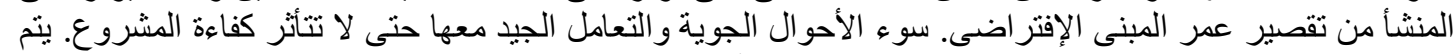

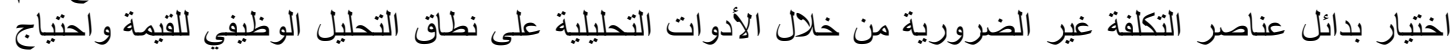

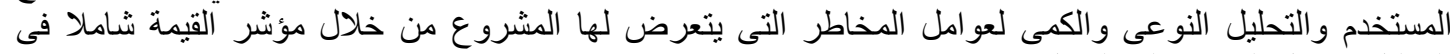

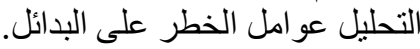

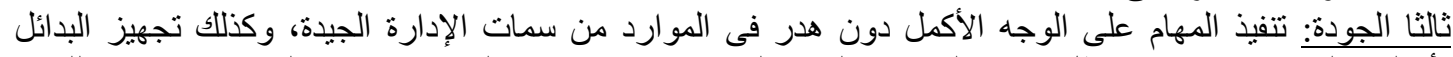

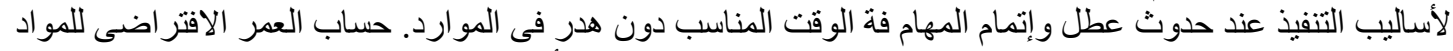

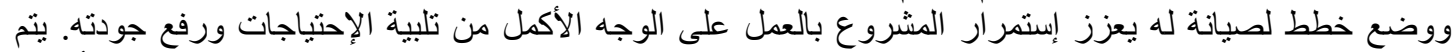

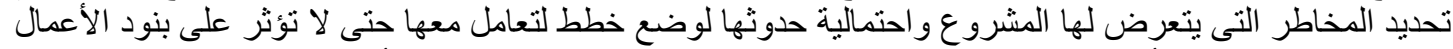

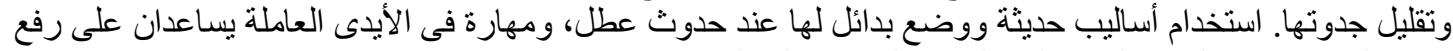

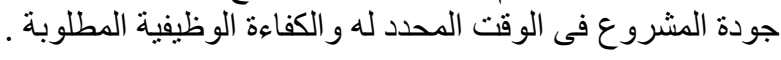


تكامل إدارة القيمة وتحليل المخاطر بمشروعات التشييد والبناء.

\begin{tabular}{|c|c|c|c|c|c|c|c|c|c|c|c|c|c|c|c|c|c|c|c|c|c|}
\hline \multirow[b]{2}{*}{ 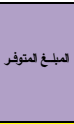 } & \multirow{2}{*}{ 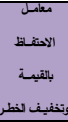 } & \multirow{2}{*}{ 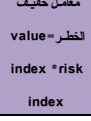 } & \multicolumn{3}{|c|}{ | عدم تتظام معدلات الآنتاج } & \multicolumn{3}{|c|}{ | عمالة غير مؤهله فيا } & \multicolumn{3}{|c|}{ | تأخر فى وصول الموزد } & \multicolumn{6}{|c|}{ 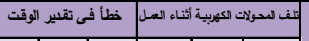 } & \multirow[b]{2}{*}{ معامل القيمة } & \multirow[b]{2}{*}{ 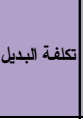 } & \multirow[b]{2}{*}{ | تكلفة البند } & \multirow[b]{2}{*}{ البديل } \\
\hline & & & 弯 & 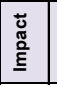 & $\begin{array}{l}\frac{2}{2} \\
\frac{2}{0} \\
\frac{0}{2} \\
\frac{0}{2} \\
\end{array}$ & 挡 & \begin{tabular}{|l|}
$\underline{\tilde{U}}$ \\
$\underline{\tilde{g}}$ \\
$\underline{\underline{\Xi}}$ \\
\end{tabular} & 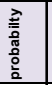 & 弯 & $\begin{array}{l}\underline{0} \\
\tilde{0} \\
\underline{\underline{0}} \\
\end{array}$ & 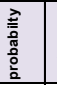 & 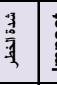 & 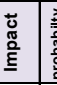 & $\begin{array}{l}\frac{2}{2} \\
\frac{\bar{z}}{0} \\
\frac{0}{2} \\
\frac{0}{2} \\
\end{array}$ & 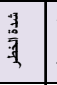 & $\begin{array}{l}\underline{\underline{z}} \\
\text { 产 } \\
\underline{\underline{E}} \\
\end{array}$ & 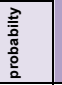 & & & & \\
\hline \multirow{3}{*}{136727.27} & \multirow{3}{*}{1.31} & \multirow{3}{*}{1.12} & $1 \%$ & $10 \%$ & $10 \%$ & $6 \%$ & $20 \%$ & $30 \%$ & $5 \%$ & $10 \%$ & $50 \%$ & \begin{tabular}{l|l}
$1 \%$ & 1
\end{tabular} & \begin{tabular}{l|l}
$10 \%$ & 1
\end{tabular} & $10 \%$ & $10 \%$ & $20 \%$ & $50 \%$ & 1.17 & 940000 & 1100000 & |بوروسلين أرضيات 60" 60 . \\
\hline & & & \multicolumn{15}{|c|}{$4.6 \%$} & \multicolumn{4}{|c|}{ متوسط تأثير شدة المخاطر على البديل } \\
\hline & & & \multicolumn{15}{|c|}{$1+(-0.046)=0.954$} & \multicolumn{4}{|c|}{ بالنسبة لمعامل القيمة Risk index } \\
\hline & & & & & & & & & & & & & & & & & & معامل القيدة & |تكلفة البديل| & | تكلفة البند & البديل \\
\hline \multirow{3}{*}{74181.82} & \multirow{3}{*}{1.07} & \multirow{3}{*}{0.99} & $12 \%$ & $40 \%$ & $30 \%$ & $6 \%$ & $20 \%$ & $30 \%$ & $10 \%$ & $20 \%$ & $50 \%$ & \begin{tabular}{l|l}
$1 \%$ & 1 \\
\end{tabular} & \begin{tabular}{l|l}
$10 \%$ & 1 \\
\end{tabular} & $10 \%$ & $10 \%$ & $20 \%$ & $50 \%$ & 1.08 & 1020000 & 1100000 & HDF أرضيات H \\
\hline & & & \multicolumn{15}{|c|}{$7.8 \%$} & \multicolumn{4}{|c|}{ متوسط تأثير المخاطر على البديل } \\
\hline & & & \multicolumn{15}{|c|}{$1+(-0.078)=0.922$} & \multicolumn{4}{|c|}{ بالنسبة لمعامل القيمة Risk index } \\
\hline
\end{tabular}

- - نلاحظ أن البديل أرضيات البورسلين • 7* * 7 سم هو الأفضل من حيث أعلى مؤشر قيمة وكذلك يوفر فى تكلفة البند

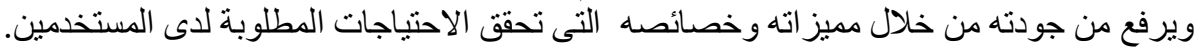

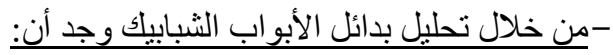

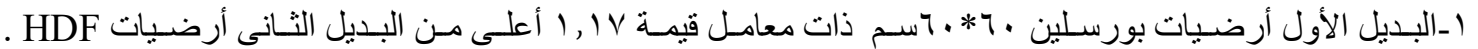

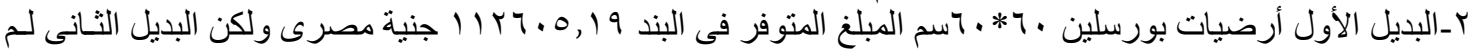

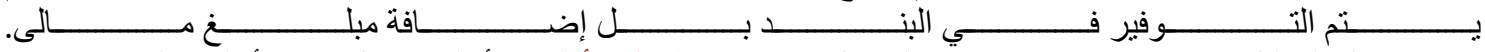

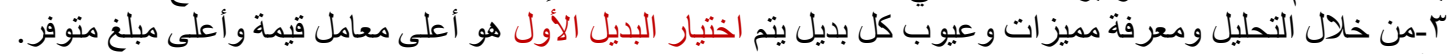

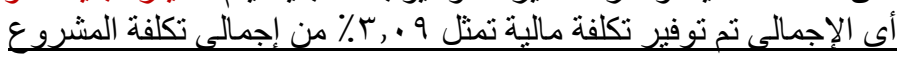

צ-Y - צ- الخطوة السادسة: التُعامل مع المخاطر شديدة الخطورة.

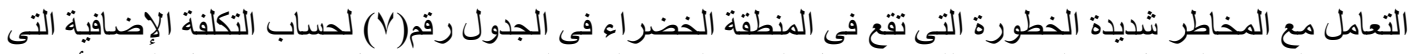

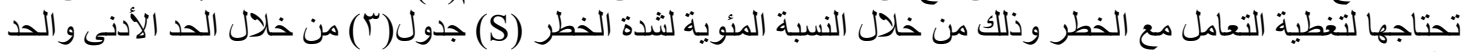
الأقصى لها ويتم تحديد نسبة القيمة الإضافية للتكلفة.

الجدول(· ( ): جدول حساب القيم الإضافية لبنود الأعمال وفقا لثدة الخطر (S).

المصدر : تحليل الباحثة.

\begin{tabular}{|c|c|c|c|c|c|c|c|c|c|c|c|c|c|c|c|c|c|c|c|c|c|}
\hline \multirow{2}{*}{ |الإضفافية } & \multicolumn{2}{|c|}{ معلات الانتاج } & \multicolumn{2}{|c|}{ عمؤلة فنيا } & \multicolumn{2}{|c|}{ علم توافر } & \multicolumn{2}{|c|}{ تأخر وصول } & \multicolumn{2}{|c|}{ سوء إلارة } & \multicolumn{2}{|c|}{ خطأ في تقيير } & \multicolumn{2}{|c|}{ المعلمى العـاد السوق } & \multicolumn{2}{|c|}{ تغير الرسومات } & \multicolumn{2}{|c|}{ الخدمات العامـة } & \multirow{2}{*}{ تكلفة البند } & \multirow{2}{*}{\multicolumn{2}{|c|}{$\begin{array}{l}\text { Risk) أعمال الخطر (Factors } \\
\text { (أبنود الأعمال }\end{array}$}} \\
\hline & الإضافية & (S) & الإضافية & (S) & |الإضافية & (S) & الإضافية & (S) & الإضافية & (S) & الإضافية & (S) & الإضافية & (S) & الإضافية & (S) & |الإضافية & (S) & & & \\
\hline 197.26 & 394.5 & $12 \%$ & 98.63 & $1 \%$ & 98.63 & $1 \%$ & 98.63 & $1 \%$ & 98.63 & $1 \%$ & 98.63 & $1 \%$ & 98.63 & $1 \%$ & 98.63 & $1 \%$ & 394.5 & $20 \%$ & 39450 & الأعمال التمهينية & \\
\hline 114709 & 10428 & $4 \%$ & 10428 & $4 \%$ & 10428 & $4 \%$ & 10428 & $1 \%$ & 10428 & $1 \%$ & 31284 & \begin{tabular}{|l|}
$6 \%$ \\
\end{tabular} & 83425 & $40 \%$ & 31284 & $6 \%$ & 41712 & $20 \%$ & 4171225 & الأعمال الخرسانية & 2 \\
\hline 24039 & 2003 & $4 \%$ & 8013 & $12 \%$ & 2003 & $1 \%$ & 6010 & $6 \%$ & 2003 & $1 \%$ & 2003 & $4 \%$ & 8013 & $10 \%$ & 16026 & $40 \%$ & 2003 & $1 \%$ & 801300 & |كعـال الحوائط & \\
\hline 34425 & 2869 & $1 \%$ & 11475 & $10 \%$ & 8606 & $6 \%$ & 11475 & $10 \%$ & 11475 & $10 \%$ & 2869 & $1 \%$ & 11475 & $20 \%$ & 22950 & $56 \%$ & 11475 & $10 \%$ & 1147500 & |ٔعمال الأرضيات & 4 \\
\hline 3062.1 & 278.4 & $4 \%$ & 1114 & $20 \%$ & 835.1 & $6 \%$ & 1114 & $20 \%$ & 1114 & $10 \%$ & 278.4 & $12 \%$ & 2227 & $56 \%$ & 835.1 & $6 \%$ & 1114 & $10 \%$ & 111350 & |عـال الرخام & 5 \\
\hline 20130 & 1830 & $3 \%$ & 1830 & $3 \%$ & 1830 & $1 \%$ & 5490 & $40 \%$ & 1830 & $1 \%$ & 7320 & $20 \%$ & 14640 & $56 \%$ & 5490 & $6 \%$ & 7320 & $10 \%$ & 732000 & |عمال النجارة + الأومني & 10 \\
\hline 11943 & 995.3 & $3 \%$ & 3981 & $20 \%$ & 995.3 & $3 \%$ & 995.3 & $3 \%$ & 995.3 & $3 \%$ & 3981 & $20 \%$ & 3981 & $10 \%$ & 7962 & $40 \%$ & 3981 & $20 \%$ & 398100 & الأعمال الصحية & 7 \\
\hline 1056.3 & 211.3 & $3 \%$ & 211.3 & $3 \%$ & 211.3 & $1 \%$ & 633.8 & $5 \%$ & 211.3 & $1 \%$ & 211.3 & $3 \%$ & 845 & $10 \%$ & 211.3 & $1 \%$ & 211.3 & $1 \%$ & 84500 & |عمال GRC +فيوتك & 8 \\
\hline 1421 & 812 & $12 \%$ & 203 & $3 \%$ & 203 & $1 \%$ & 203 & $3 \%$ & 203 & $1 \%$ & 203 & $3 \%$ & 1218 & $28 \%$ & 203 & $1 \%$ & 812 & $20 \%$ & 81200 & | عمال الحدادة & 99 \\
\hline 8360 & 4180 & $12 \%$ & 4180 & $12 \%$ & 4180 & $10 \%$ & 4180 & $10 \%$ & 4180 & $10 \%$ & 4180 & $20 \%$ & 4180 & $10 \%$ & 4180 & $20 \%$ & 3135 & $6 \%$ & 417950 & | أعمال الكهرباء & 1 \\
\hline 595.1 & \begin{tabular}{|l|}
148.8 \\
\end{tabular} & $1 \%$ & 148.8 & $3 \%$ & 148.8 & $3 \%$ & 148.8 & $3 \%$ & 148.8 & $3 \%$ & 148.8 & \begin{tabular}{|l|}
$1 \%$ \\
\end{tabular} & 446.3 & $6 \%$ & 148.8 & $1 \%$ & \begin{tabular}{|l|l|}
148.8 \\
\end{tabular} & $1 \%$ & 59500 & |عمال مكملة & $\#$ \\
\hline 522 & 261 & $20 \%$ & 65.25 & $1 \%$ & 261 & $10 \%$ & 261 & $20 \%$ & 261 & $10 \%$ & 261 & $20 \%$ & 261 & $10 \%$ & 261 & $10 \%$ & 65.25 & $1 \%$ & 6100 & أعمال الموقع العام & \\
\hline \multirow[t]{2}{*}{220460} & & & & & & & & & & & & & & & & & & & 8070175 & إجمالى تكلفة المشروع & \\
\hline & & $7 \%$ & & $8 \%$ & & $4 \%$ & & $10 \%$ & & $4 \%$ & & $9 \%$ & & $21 \%$ & & $16 \%$ & & $10 \%$ & \multicolumn{3}{|c|}{ متوسط نسبة شدة } \\
\hline
\end{tabular}

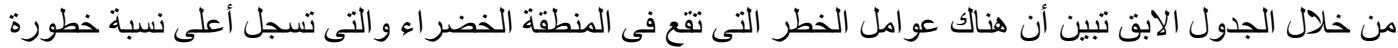
و هى(تغير الرسومات أثناء العمل- اعتماد السوق المحلى على الواردات الأجنبية) وقيم التكلفة الإضافية لكل عامل خطر التهر 
تكامل إدارة القيمة وتحليل المخاطر بمشروعات التشييد والبناء.

- من التحليل القيمي للقيمة تبين أن مواطن التكلفة غير الضرورية تكمن في الأعمال الإنشائية(الأعمال الخرسانية)

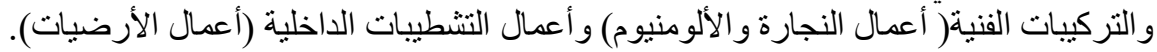

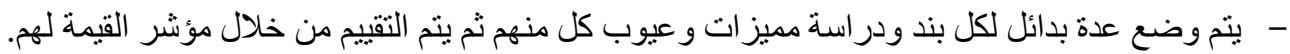

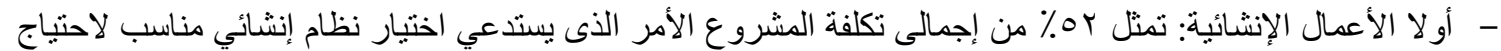

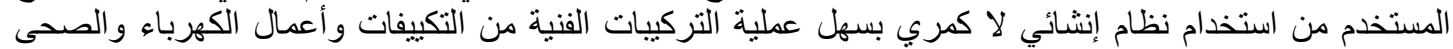

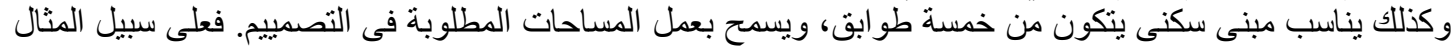

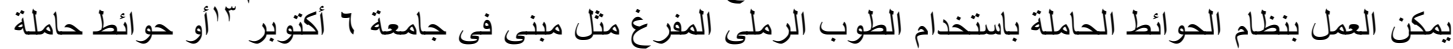

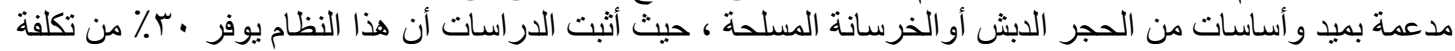

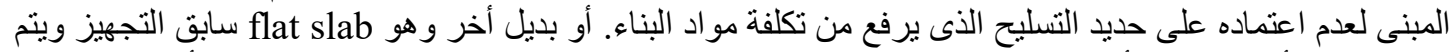

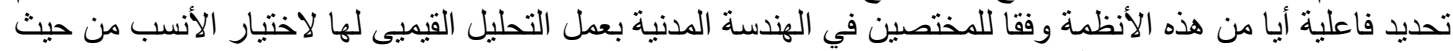

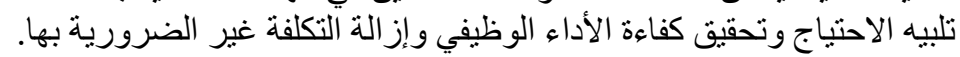

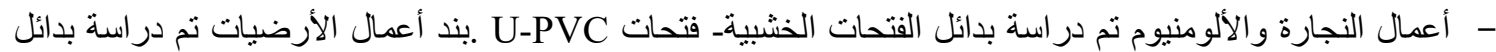
أرضيات البورسلين - أرضيات HDF.

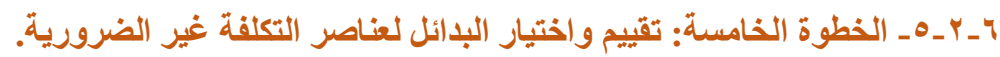

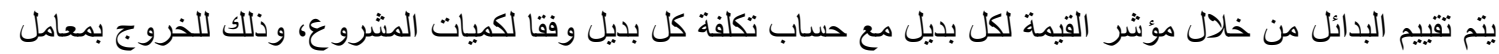

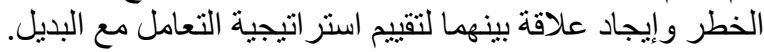
الجدول(^): تقييم واختيار بدائل أعمال الأبواب و الثبابيك. المصدر : تحليل الباحثة.

\begin{tabular}{|c|c|c|c|c|c|c|c|c|c|c|c|c|c|c|c|c|c|c|c|c|c|}
\hline \multirow[b]{2}{*}{ | البلغ التوفر } & \multirow{2}{*}{ وتخثيف لنظر } & \multirow{2}{*}{ 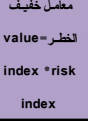 } & \multicolumn{3}{|c|}{ | عدم تنظمام معدلات الاتتاج } & \multicolumn{3}{|c|}{ كالة غير مؤهله فيا } & \multicolumn{3}{|c|}{ | تأذر فى وصول المواد } & \multicolumn{3}{|c|}{ | خطا في تقير الوقت } & \multicolumn{3}{|c|}{ 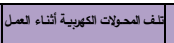 } & \multirow[b]{2}{*}{$\begin{array}{c}\text { معامل القيدة } \\
\text { value index }\end{array}$} & \multirow[b]{2}{*}{ تكلفة البديل | } & \multirow[b]{2}{*}{ ت تكلفة البند } & \multirow[b]{2}{*}{ البديل } \\
\hline & & & 草 & 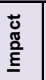 & 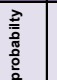 & 竞 & \begin{tabular}{|l|}
$\underline{\bar{\sigma}}$ \\
$\underline{\underline{\sigma}}$ \\
$\underline{\underline{\varepsilon}}$
\end{tabular} & 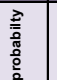 & 亲 & \begin{tabular}{|l|}
$\underline{\tilde{U}}$ \\
$\underline{\underline{\sigma}}$ \\
$\underline{\underline{E}}$
\end{tabular} & 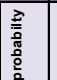 & 亲 & $\begin{array}{l}\overline{\mathrm{u}} \\
\underline{\underline{\underline{\sigma}}} \\
\underline{\underline{E}}\end{array}$ & 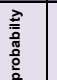 & 亲 & 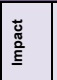 & 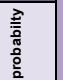 & & & & \\
\hline \multirow{3}{*}{112605.19} & \multirow{3}{*}{1.39} & \multirow{3}{*}{1.12} & $12 \%$ & $40 \%$ & $30 \%$ & $6 \%$ & $20 \%$ & $30 \%$ & $5 \%$ & $\mid 10 \%$ & $50 \%$ & $10 \%$ & $20 \%[5$ & $50 \%$ & $10 \%$ & $20 \%$ & $50 \%$ & 1.23 & 593000 & 732000 & U-PVC إبواب وشبابيك U \\
\hline & & & \multicolumn{15}{|c|}{$8.6 \%$} & \multicolumn{4}{|c|}{ متوسط تأثير شدة المخاطر على البديل } \\
\hline & & & \multicolumn{15}{|c|}{$1+(-0.086)=0.91$} & \multicolumn{4}{|c|}{ بالنسبة لمعامل القيمة Risk index } \\
\hline & & & & & & & & & & & & & & & & & & $\begin{array}{c}\text { معلمل القيدة } \\
\text { value index }\end{array}$ & | تكلفة البديل | & | تكلفة البند & البديل \\
\hline \multirow{3}{*}{-114935.79} & \multirow{3}{*}{0.60} & \multirow{3}{*}{0.68} & $20 \%$ & $40 \%$ & \begin{tabular}{|l|l|}
$50 \%$ & \\
\end{tabular} & $40 \%$ & $80 \%$ & $50 \%$ & $40 \%$ & $80 \%$ & $50 \%$ & $3 \%$ & $10 \%$ & \begin{tabular}{|l|l|}
$30 \%$ \\
\end{tabular} & $10 \%$ & $20 \%$ & $50 \%$ & 0.88 & 833000 & 732000 & أبواب وشبابيك خثب كونتر \\
\hline & & & \multicolumn{15}{|c|}{$22.6 \%$} & \multicolumn{4}{|c|}{ متوسط تأثير المخاطر على البديل } \\
\hline & & & \multicolumn{15}{|c|}{$1+(-0.226)=0.774$} & \multicolumn{4}{|c|}{ Risk index بالنسبة لمعامل القيـة } \\
\hline
\end{tabular}

- - نلاحظ أن البديل Uو الأفضل من حيث أعلى مؤشر قيمة وكذلك يوفر فى تكلفة البند ويرفع من جودته من

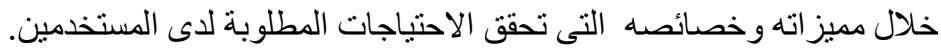

من خلال تحليل بدائل الأبو ابو الثباييك وجد أن:

ـ ـ البديل الأول أبو اب وشبابيك U-PVC ذات معامل قيمة سب , 1 أعلى من البديل الثانى أبو اب وشبابيك خشب كونتر.

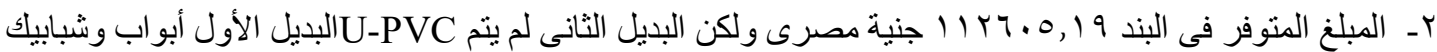
التوفير في البند بل إضافة مبلغ مالى. rـ من خلال التحليل ومعرفة مميز ات وعيوب كل بديل يتم اختيار البديل الأول هو أعلى معامل قيمة و أعلى مبلغ منوفر . الجدول(9): تقييم واختيار بدائل أعمال الأرضيات.

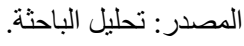


تكامل إدارة القيمة وتحليل المخاطر بمشروعات التشييد والبناء.

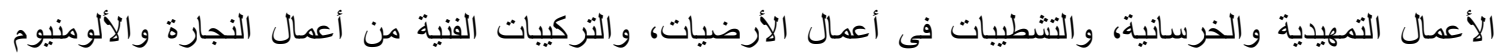
و الأعمال الصحية و أعمال GRC و الفية والفيوتلك وأعمال الكهرباء.

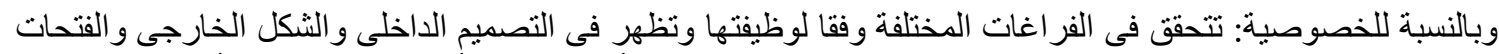

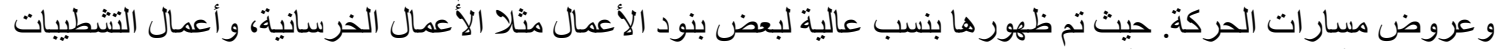
الداخلية من أعمال المبانى و الأرضيات و التركيبات الفنية بنسب منوسطة.

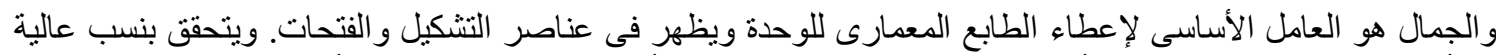
فى أعمال التشطيبات الداخلية من أعمال الرخام و التركيبات الفنية من أعمال GRC و الفيوتلك، و وأعمال الموقع العام. ولإل وبنسب

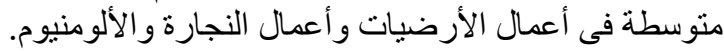

ويتم التعبير عن الجودة بإظهار الجانب الوظيفي و الجمالى و التميز فى كل عنصر بالوحدة وهو يكمن فى جميع بنود الاعمال بنسب متوسطة.

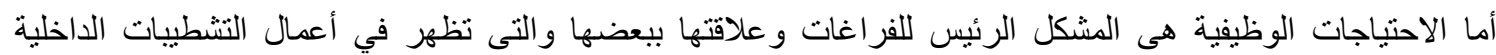
و الخارجية من أعمال الحو ائط و الرخام بنسب عالية وأعمال التركيبات الفنبة و الأرضيات بنسب متوسطة.

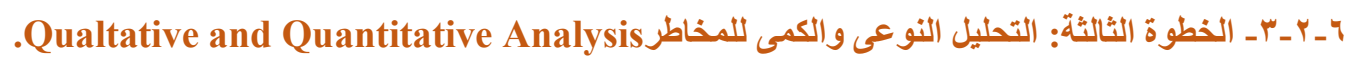
من خلال التحليل النو عى لفريق العمل لعو امل المخاطر التى يتعرض لهال الها المشروع على على عناصر التحليل يتم حساب القيمة

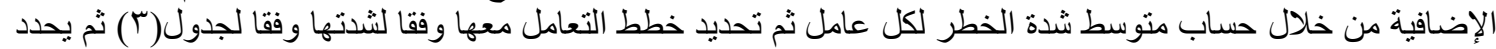

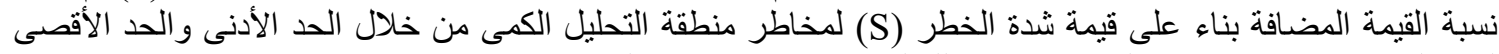

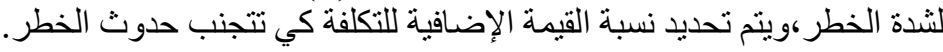

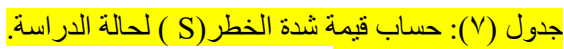

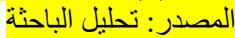

\begin{tabular}{|c|c|c|c|c|c|c|c|c|c|c|c|c|c|c|c|c|c|c|c|c|c|c|c|c|c|c|c|c|c|}
\hline \multicolumn{3}{|c|}{ علم إنتظام معدلات } & \multicolumn{3}{|c|}{ عمالة غير مؤهلة فنيا } & \multicolumn{3}{|c|}{ عدم توافر المعدات } & \multicolumn{3}{|c|}{ ل مل المواد } & \multicolumn{3}{|c|}{ المشروع } & \multicolumn{3}{|c|}{ خطأ في تقدير الوقت } & \multicolumn{3}{|c|}{ على المواددات الإنبية المحلى } & \multicolumn{3}{|c|}{ تغير الرسومات أثناء } & \multicolumn{3}{|c|}{ علعم توافر الخدمات } & \multirow{2}{*}{ | تكلفة البند } & \multicolumn{2}{|l|}{$\begin{array}{l}\text { أعمال الخطر(Fisk } \\
\text { (Factors }\end{array}$} \\
\hline (S) & (1) & (p) & (S) & (1) & (p) & (S) & (1) & (p) & (S) & (1) & (p) & (S) & (1) & (p) & (S) & (1) & (p) & (S) & (1) & (p) & (S) & (1) & $(p)$ & (S) & (1) & (p) & & & \\
\hline $12 \%$ & $40 \%$ & $30 \%$ & $1 \%$ & $10 \%$ & $10 \%$ & $1 \%$ & $10 \%$ & $10 \%$ & $1 \%$ & $10 \%$ & $10 \%$ & $1 \%$ & $10 \%$ & $10 \%$ & $1 \%$ & $10 \%$ & $10 \%$ & $1 \%$ & $10 \%$ & $10 \%$ & $1 \%$ & $10 \%$ & $10 \%$ & $20 \%$ & $40 \%$ & $50 \%$ & 39450 & |لأعمال التقيلية & \\
\hline $4 \%$ & $40 \%$ & $10 \%$ & $4 \%$ & $40 \%$ & $10 \%$ & $4 \%$ & $40 \%$ & $10 \%$ & $1 \%$ & $10 \%$ & $10 \%$ & $1 \%$ & $10 \%$ & $10 \%$ & $6 \%$ & $20 \%$ & $30 \%$ & $40 \%$ & $80 \%$ & $50 \%$ & $6 \%$ & $20 \%$ & $30 \%$ & $20 \%$ & \begin{tabular}{l|l}
$40 \%$ & 5
\end{tabular} & $50 \%$ & \begin{tabular}{|l|l|l|l|l|}
41725 \\
\end{tabular} & |الأعمال الخرسانية & 2 \\
\hline $4 \%$ & $40 \%$ & $10 \%$ & $12 \%$ & $40 \%$ & $30 \%$ & $1 \%$ & $10 \%$ & $10 \%$ & $6 \%$ & $20 \%$ & $30 \%$ & $1 \%$ & $10 \%$ & $10 \%$ & $4 \%$ & $40 \%$ & $10 \%$ & $10 \%$ & $20 \%$ & $50 \%$ & $40 \%$ & $80 \%$ & $50 \%$ & $1 \%$ & $10 \%$ & $10 \%$ & 801300 & | أعمال الحوائط & \\
\hline $1 \%$ & $10 \%$ & $10 \%$ & $10 \%$ & $20 \%$ & $50 \%$ & $6 \%$ & $20 \%$ & $30 \%$ & $10 \%$ & $20 \%$ & $50 \%$ & $10 \%$ & $20 \%$ & $50 \%$ & $1 \%$ & $10 \%$ & $10 \%$ & $20 \%$ & $40 \%$ & $50 \%$ & $56 \%$ & $80 \%$ & $70 \%$ & $10 \%$ & $20 \%$ & $50 \%$ & $\mid 1147500$ & |ٔعمال الأرضيات & 4 \\
\hline $4 \%$ & $40 \%$ & $10 \%$ & $20 \%$ & $40 \%$ & $50 \%$ & $6 \%$ & $20 \%$ & $30 \%$ & $20 \%$ & $40 \%$ & $50 \%$ & $10 \%$ & $20 \%$ & $50 \%$ & $12 \%$ & $40 \%$ & $30 \%$ & $56 \%$ & $80 \%$ & $70 \%$ & $6 \%$ & $20 \%$ & $30 \%$ & $0 \%$ & $20 \%$ & $50 \%$ & 111350 & | أعمال الرخام & 3 \\
\hline $3 \%$ & $10 \%$ & $30 \%$ & $3 \%$ & $10 \%$ & $30 \%$ & $1 \%$ & $10 \%$ & $10 \%$ & $40 \%$ & $80 \%$ & $50 \%$ & $1 \%$ & $10 \%$ & $10 \%$ & $20 \%$ & $40 \%$ & $50 \%$ & $56 \%$ & $80 \%$ & $70 \%$ & $6 \%$ & $20 \%$ & $30 \%$ & $10 \%$ & $20 \%$ & $50 \%$ & 732000 & أعمال النجارة + الألومن & 6 \\
\hline $3 \%$ & $10 \%$ & $30 \%$ & $20 \%$ & $40 \%$ & $50 \%$ & $3 \%$ & $10 \%$ & $30 \%$ & $3 \%$ & $10 \%$ & $30 \%$ & $3 \%$ & $10 \%$ & $30 \%$ & $20 \%$ & $40 \%$ & $50 \%$ & $10 \%$ & $20 \%$ & $50 \%$ & $40 \%$ & $80 \%$ & \begin{tabular}{|l|l|}
$50 \%$ \\
\end{tabular} & $20 \%$ & $40 \%$ & $50 \%$ & 398100 & |لألعمال الصية |لإية & 7 \\
\hline $3 \%$ & $10 \%$ & $30 \%$ & $3 \%$ & $10 \%$ & $30 \%$ & $1 \%$ & $10 \%$ & $10 \%$ & $5 \%$ & $10 \%$ & $50 \%$ & $1 \%$ & $10 \%$ & $10 \%$ & $3 \%$ & $10 \%$ & $30 \%$ & $10 \%$ & $20 \%$ & $50 \%$ & $1 \%$ & $10 \%$ & $10 \%$ & $1 \%$ & $10 \%$ & $10 \%$ & 84500 & أعمال GRC +فيوتك & 8 \\
\hline $12 \%$ & $40 \%$ & $30 \%$ & $3 \%$ & $10 \%$ & $30 \%$ & $1 \%$ & $10 \%$ & $10 \%$ & $3 \%$ & $10 \%$ & $30 \%$ & $1 \%$ & $10 \%$ & $10 \%$ & $3 \%$ & $10 \%$ & $30 \%$ & $28 \%$ & $40 \%$ & $70 \%$ & $1 \%$ & $10 \%$ & $10 \%$ & $20 \%$ & $40 \%$ & $50 \%$ & 81200 & | & 9 \\
\hline $12 \%$ & $40 \%$ & $30 \%$ & $12 \%$ & $40 \%$ & $30 \%$ & $10 \%$ & $20 \%$ & $50 \%$ & $10 \%$ & $20 \%$ & $50 \%$ & $10 \%$ & $20 \%$ & $50 \%$ & $20 \%$ & \begin{tabular}{|l|l|}
$40 \%$ & \\
\end{tabular} & $50 \%$ & $10 \%$ & $20 \%$ & $50 \%$ & $20 \%$ & $40 \%$ & $50 \%$ & $6 \%$ & $20 \%$ & $30 \%$ & 417950 & | & 10 \\
\hline $1 \%$ & $10 \%$ & $10 \%$ & $3 \%$ & $10 \%$ & $30 \%$ & $3 \%$ & $10 \%$ & $30 \%$ & $3 \%$ & $10 \%$ & $30 \%$ & $3 \%$ & $10 \%$ & $30 \%$ & $1 \%$ & $10 \%$ & $10 \%$ & $6 \%$ & $20 \%$ & $30 \%$ & $1 \%$ & $10 \%$ & $10 \%$ & $1 \%$ & $10 \%$ & $10 \%$ & 59500 & |cعال مكملة & 11 \\
\hline $20 \%$ & $40 \%$ & $50 \%$ & $1 \%$ & $10 \%$ & $10 \%$ & $10 \%$ & $20 \%$ & $50 \%$ & $20 \%$ & $40 \%$ & $50 \%$ & $10 \%$ & $20 \%$ & $50 \%$ & $20 \%$ & $40 \%$ & $50 \%$ & $10 \%$ & $20 \%$ & $50 \%$ & $10 \%$ & $20 \%$ & $50 \%$ & $1 \%$ & $10 \%$ & $10 \%$ & 26100 & |أعمال الموقع العام & 12 \\
\hline & & & & & & & & & & & & & & & & & & & & & & & & & & & \begin{tabular}{|l|l|}
8070175 &
\end{tabular} & إجمالى تكلفة الششروع & \\
\hline $7 \%$ & & & $8 \%$ & & & $4 \%$ & & & $10 \%$ & & & $4 \%$ & & & $9 \%$ & & & $21 \%$ & & & $16 \%$ & & & $10 \%$ & & & & متوسط نسبة شد: & \\
\hline
\end{tabular}

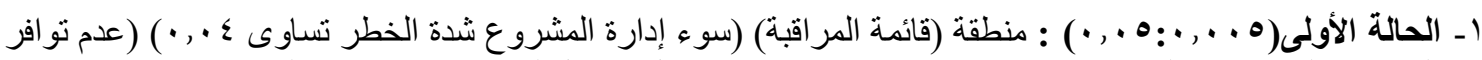

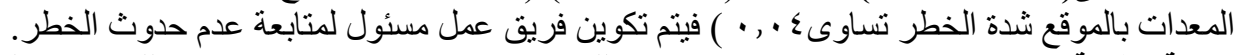

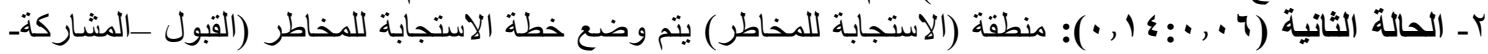

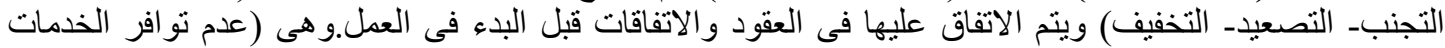

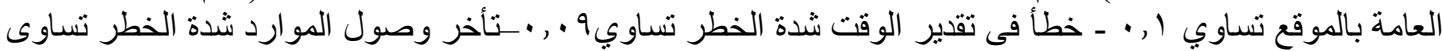

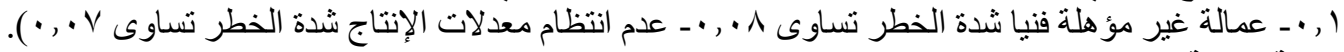

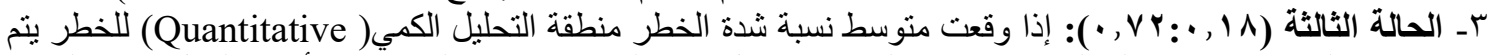

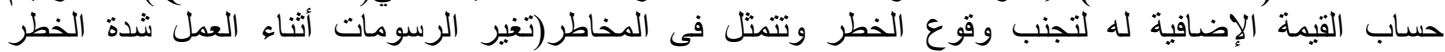

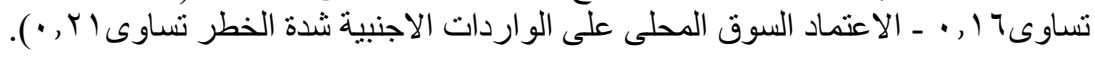

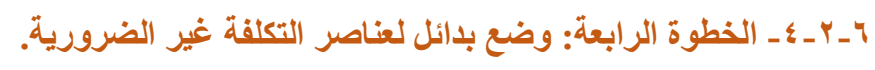


تكامل إدارة القيمة وتحليل المخاطر بمشروعات التشييد والبناء.

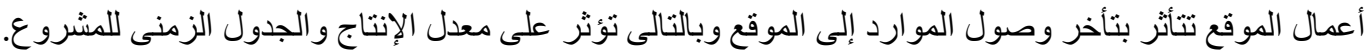

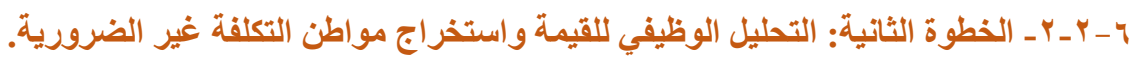

من خلال مقارنة نسب الاحتياج للمستخدمين بنسب احتباج فريق العمل و الخروج بالاحتياجات التى بها قيم سالبة هى التى التى التي

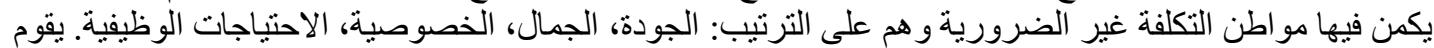

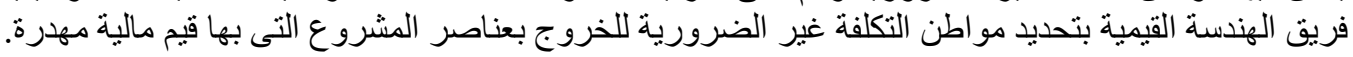
جدول (7): التحلبل الوظيفي للقيمة لاستخر اج عناصر التكلفة غبر الضرورية لحالة الدراسة. المصدر : تحليل الباحثة

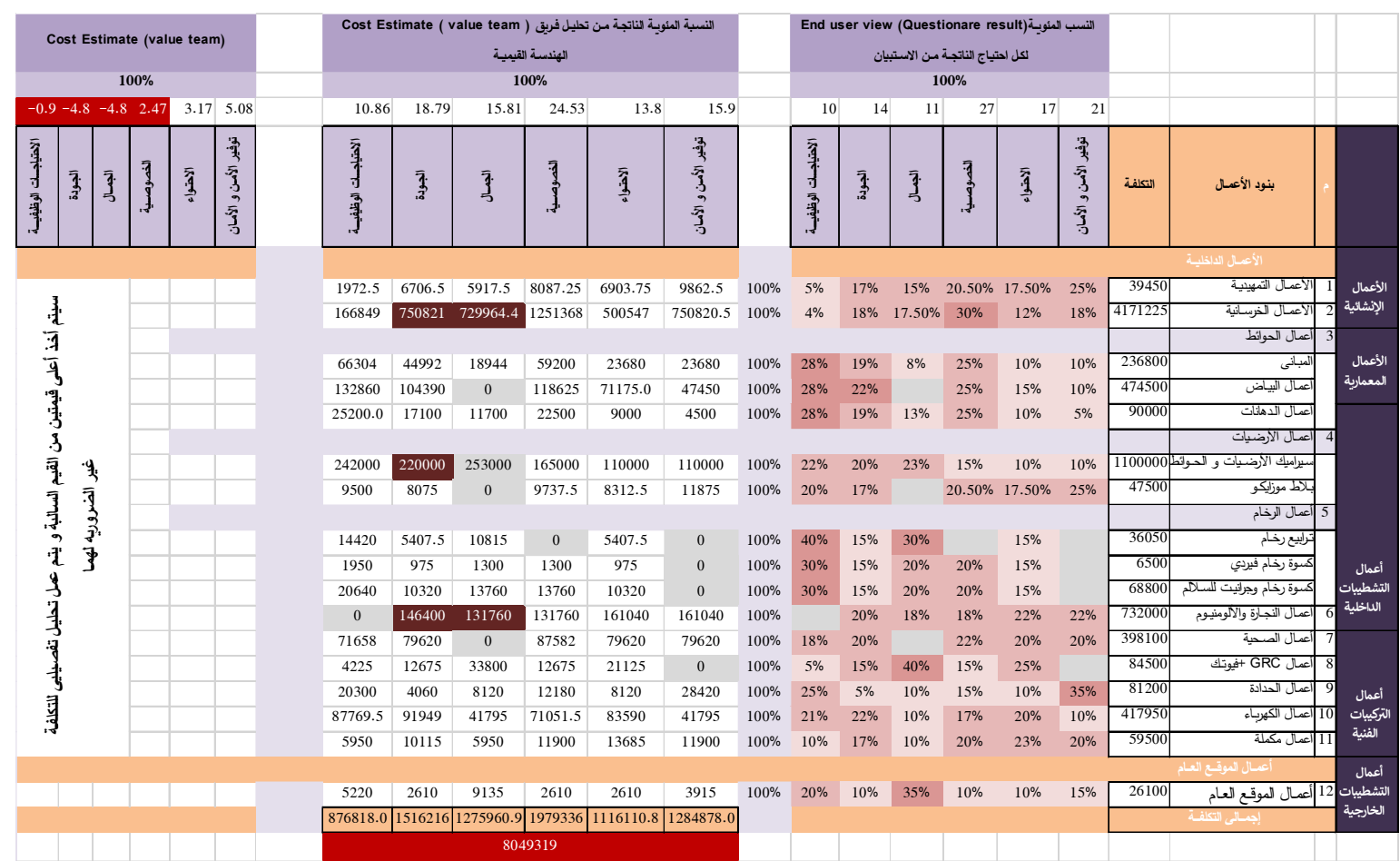

- من خلال تحليل الجدول(†) سيتم التعامل مع الاحتياجين الأعلى قيمة وهم الجمال

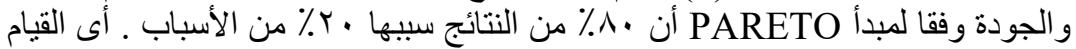

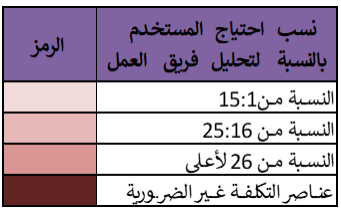

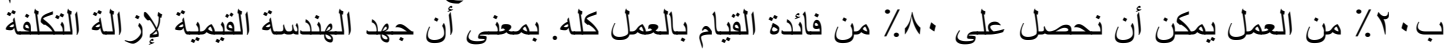

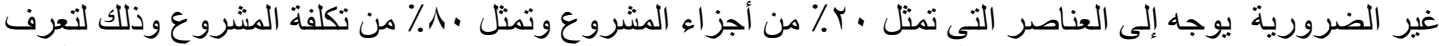

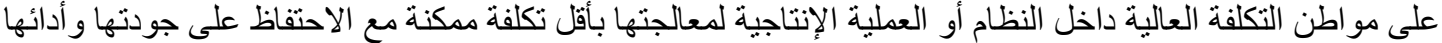

الوظيفي.

يبين التحليل الوظيفي لفريق العمل وفقا لاحتياجات المستخدم كالاتي:

أن نسبة توفير الأمن والامان: تظهر فى محددات الفراغ الأفقية والر أسية وتظهر فى التشطييات الداخلية و أعمال التركيبات

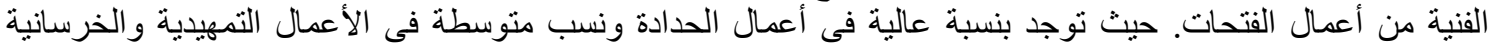

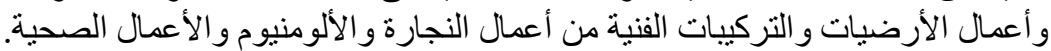

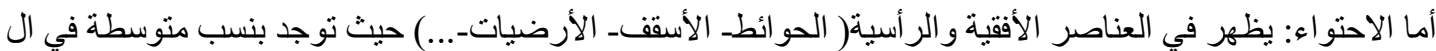


تكامل إدارة القيمة وتحليل المخاطر بمشروعات التشييد والبناء.

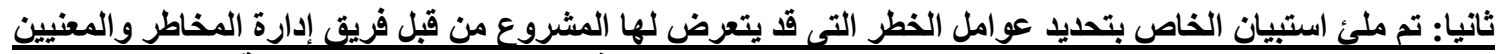

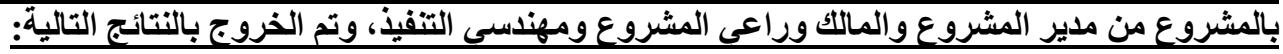

المخاطر التي تعرض لها مشروع دار مصر إسكان اجتماعي منوسط بمدينة العبور :

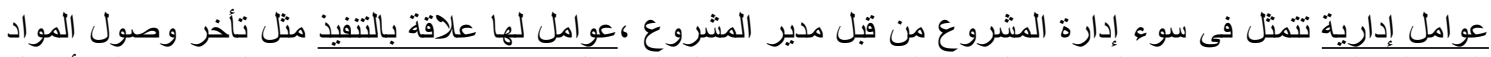

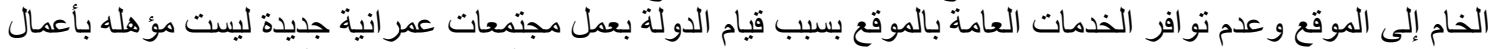

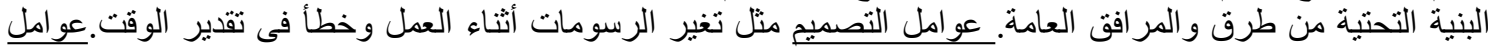

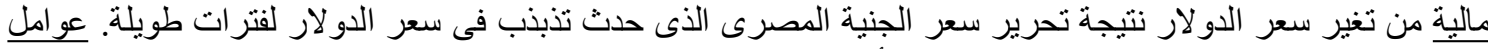

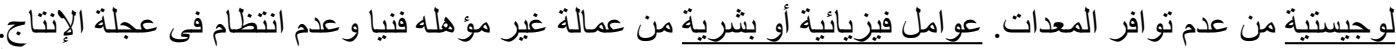

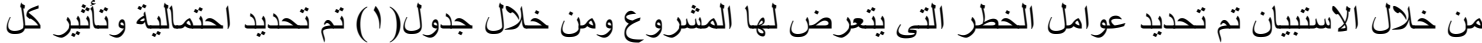

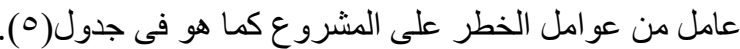

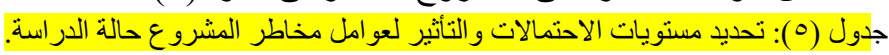

المصدر : تحليل الباحثة.

من خلال تحليل نتائج استبيان المخاطر التى يتعرض لها المشروع من خلال (الاستثارى- مهندسى التفيذ- المالكـ-

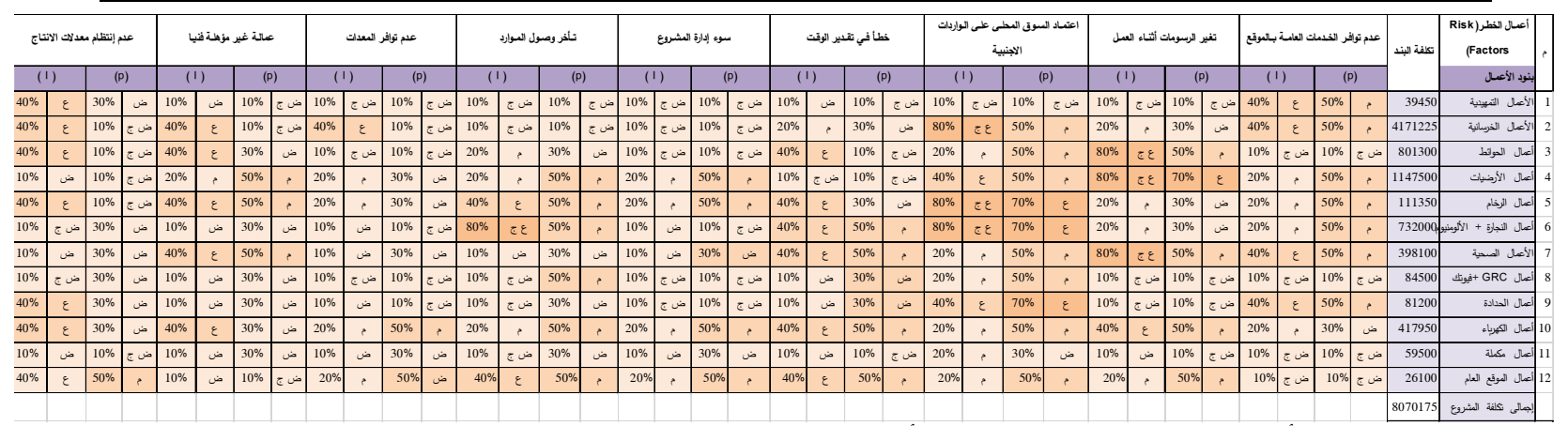

المقاول) وتحديد أثرها على بنود المشروع تبين الأتى:

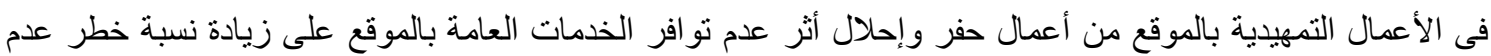

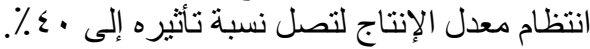

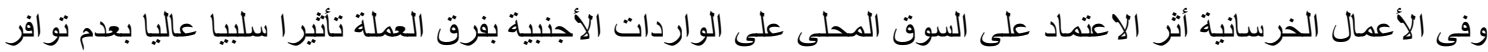

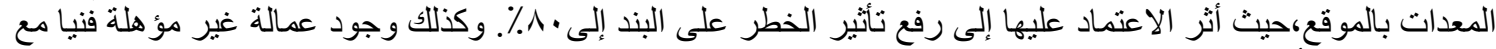

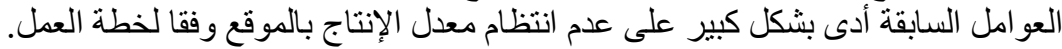

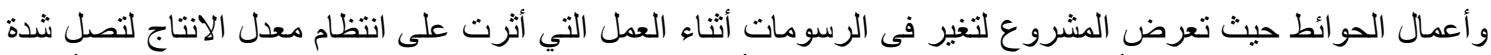

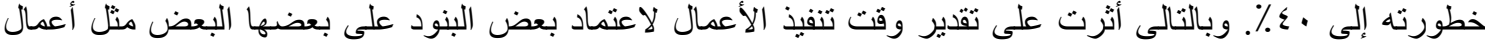

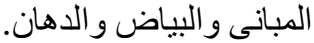

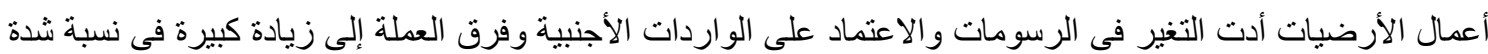

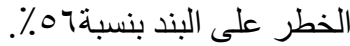
أعمال الرخام أثر الاعتماد على الواردات الاجنبية على تأخر وصول المو ارد وبالتالى على عدم انتظام الإنتاج وتأخر وقت

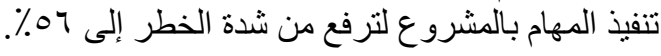
أعمال النجارة و الألومنيوم تسبب اعتماد السوق المحلى على الو اردات الأجنبية بشكل كبير الذى أدى ألى إلى ارتفاع شدة الخطر

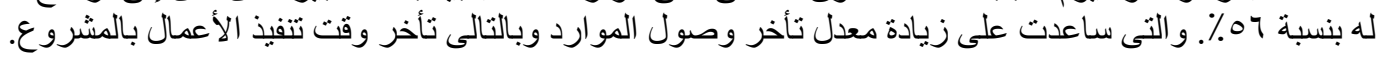

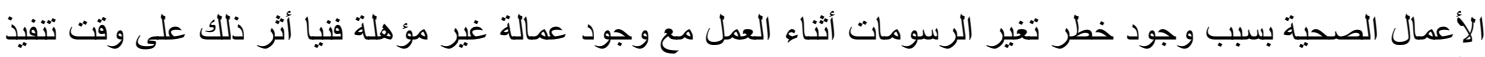

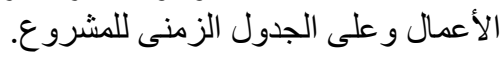

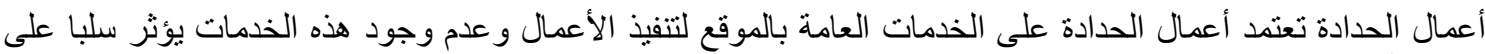
عو امل أخرى مثل عدم انتظام معدل الإنتاج.

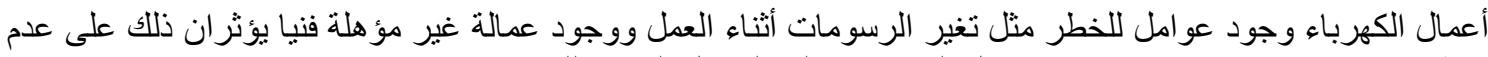

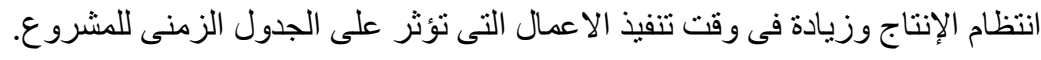


تكامل إدارة القيمة وتحليل المخاطر بمشروعات التشييد والبناء.

خرسانى. باصفات المشروع : يتكون من دور أرضي وخمسة أدوار متكررة ويوجد به أسانسير ويحيط بالمشروع سور

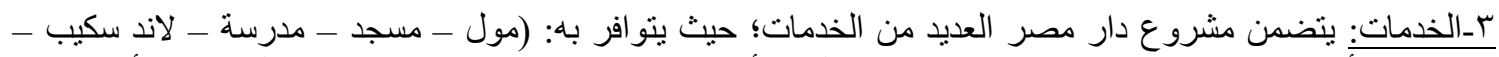
مصاعد - أسواق - محلات تجارية - خدمات منفصلة - أمن وحراسة - سور خرسانى يحيط بالمشروع (أي كمبوند

مغلق).

צ-Y - تطبيق منهجية التكامل بين الهناسة القيمية و إدارة المخاطر على مشروع دار مصر بمدينة العبور.

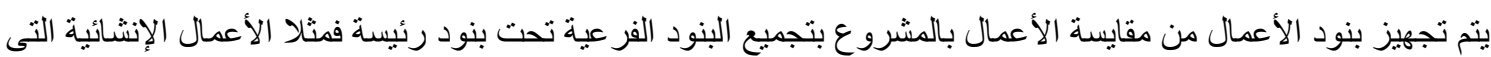

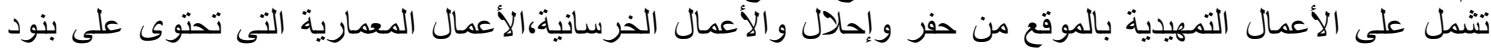

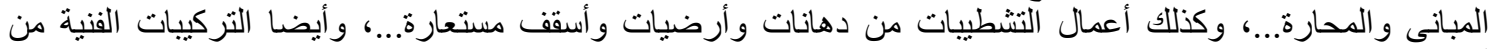
أعمال الحدادة و النجارة و الصحى و الكهرباء.

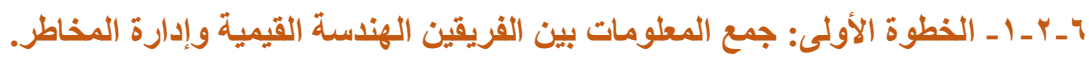

توفير فريق للهندسة القيمية و إدارة المخاطر فى المشروع من بداية العمل وينت عمل جلسات اجتماعية بينهما للمعرفة الجيدة

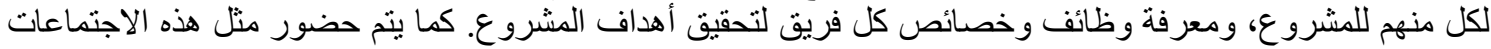
المعنيين بالمشروع مثل المالك و الاستشارى ومهندئنسي التنفيذ.

أولا: تم ملئ استبيان الخاص بتحليد احتياجات المستخدم من خلايل فريقي الهندسة القيمية وإدارة المخاطر وكذلك عينة

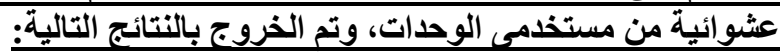

من خلال الاستبيان تم تحديد احتياجات المستخدم ومعرفة فريق العمل بها ليتم حساب الوزن النسبى لها وفقا لتكلفـة ونسبة

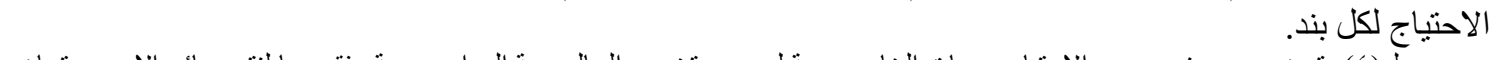

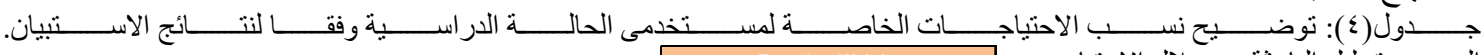

\begin{tabular}{|c|c|}
\hline & on Weight \\
\hline النسبة & الالاثباجات \\
\hline $27 \%$ & 1 |توفير الأمن والأمسان \\
\hline $17 \%$ & 2 \\
\hline $24 \%$ & 3 \\
\hline $8 \%$ & 4 \\
\hline $14 \%$ & 5 \\
\hline $10 \%$ & 6 \\
\hline $100 \%$ & \\
\hline
\end{tabular}
المصدر : تحليل الباحثة من خلال الاستبان .

ومن الاستبيان تم تحديد المواصفات والاحتياجات التصميمية للمسكن وتم الخروج بالنتائج التالية:

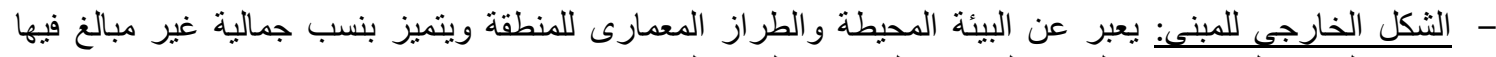

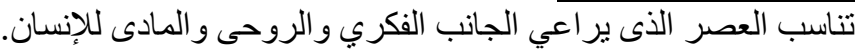

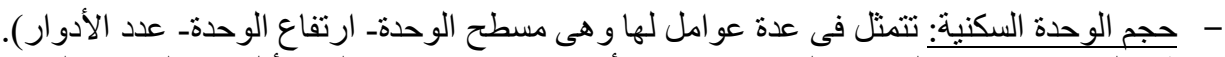

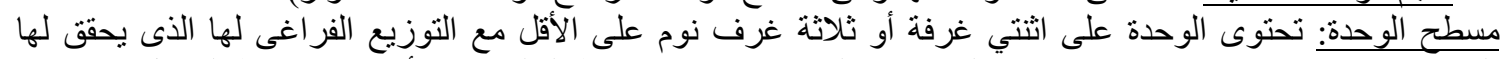

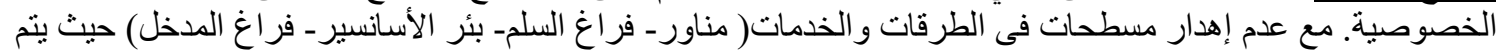

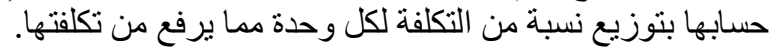

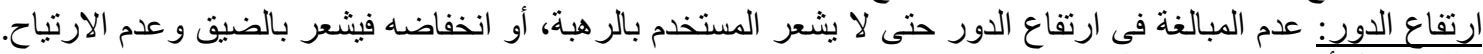

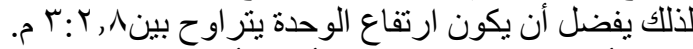

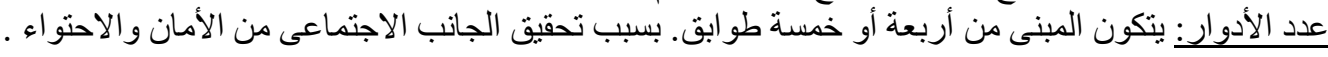

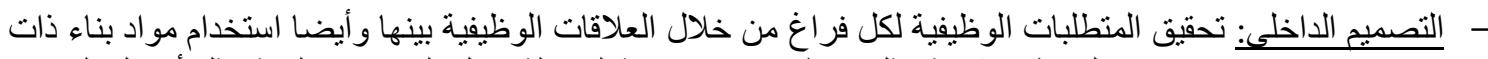
عمر افتر اضي كبير. وتفضيل نظم إنشائية مثل بلاطات غير كمرية لسهولة عمل التركيبات الفنية مثل أعمال التكييف و الكهرباعو..........

- متطلبات الحركة: عدم هدر مسطحات فى مسار ات الحركة وتحقيق الحد الادنى منها حتى لا تزيد من التكلفة للوحدة فى مسطحات غير مستغلة.

- الفتحات: استخدام نوعية فتحات لا تتأثر بالعوامل الجوية وتسريب الأثربة و المطر داخل الفراغ ، وذات أمان عال

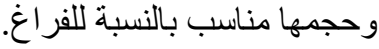
- - الخدمات: توفير أسانسير بالوحدة السكنية - توفير مدارس- مستشفياتــ مر اكز تجارية بالقرب من الوحدات. 
تكامل إدارة القيمة وتحليل المخاطر بمشروعات التشييد والبناء.

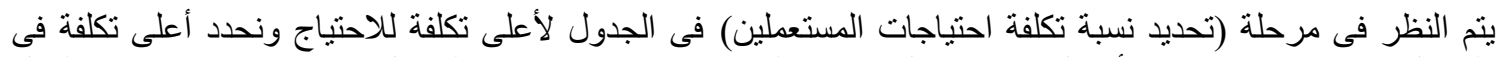

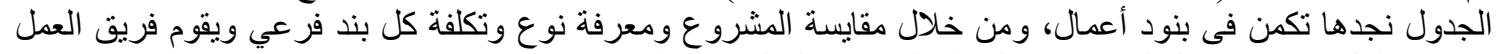

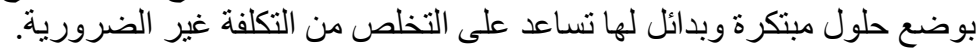

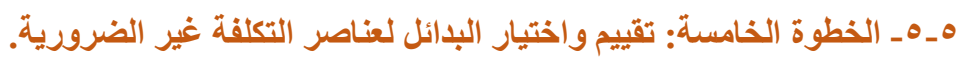

يتم تقييم البدائل لاختيار أفضلهم عن طريق عدة خطوات حسابية وهى:

- مؤشر القيمة= تكلفة البند قبل النطوير / تكلفة البند بعد التطوير

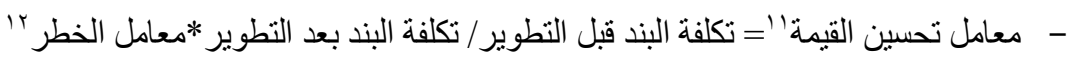

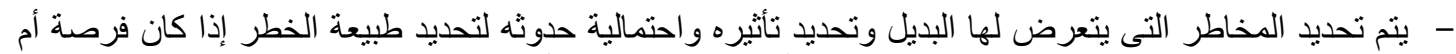

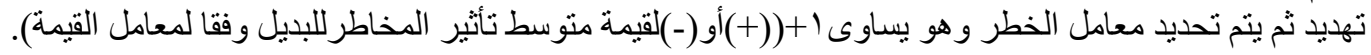

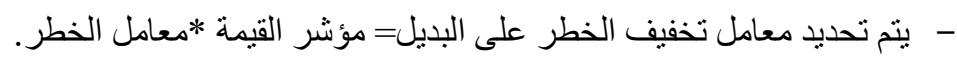

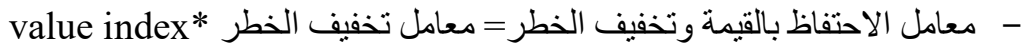

حساب القيمة المتوفرة للبديل وبدائل تطويره =(معامل تخفيف الخطر/ معامل الاحتفاظ بالقيمة وتخفيف

المخاطر)*تكلفة المتوفر من البديل.

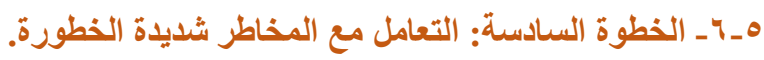

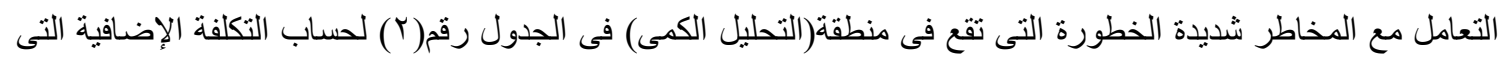

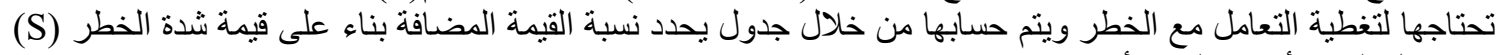

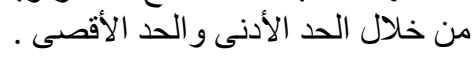

الجدول(؟): جدول تعيين نسبة القيمة الإضافية للنكلفة من خلال شدة الخطر وتكلفة البند.

\begin{tabular}{|l|r}
\hline Add. & \\
\cline { 2 - 2 } Cost & $\mathrm{Ma}$ \\
\hline $2.0 \%$ & $100 !$ \\
\hline $1.5 \%$ & $40 \%$ \\
\hline
\end{tabular}

Project Management Istitute (Guide to the project Management Body of المصندر)

Knowledge )(Newtown square . Pennsylvania,USA 2017 6thEd)p.408

צ- حالة الدراسة (مشروع دار مصر بمدينة العبور (إسكان متوسط نموذجA منفصل · م عمارات)).

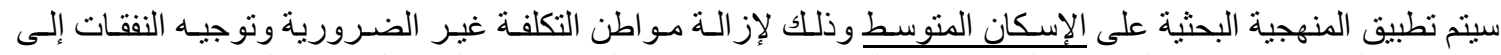

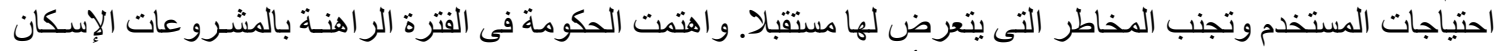

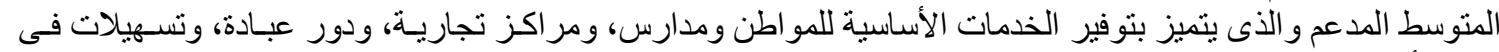
السداد أقساط تتناسب مع الفئات المجتمعية فى كل إسكان. وسبب اختبار عينة الدر اسة لنوع الإسكان المنوسط هى:

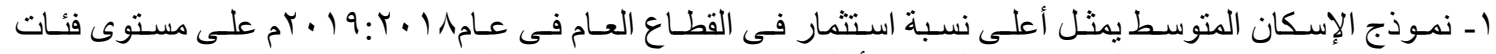

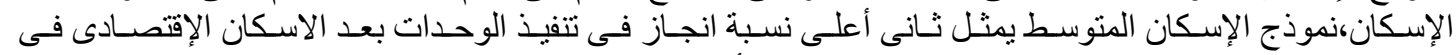

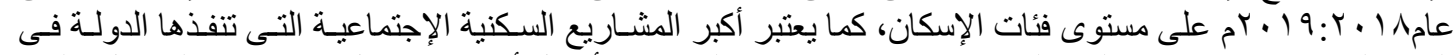

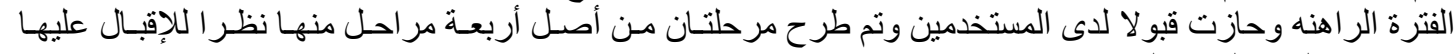

وتسهيلات السداد لسعر الوحدة.

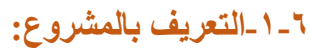

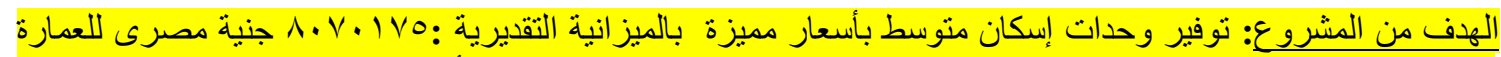

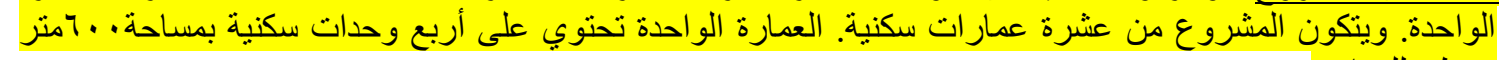

مسطح للعمارة.

1 ا-مساحات المشروع : مساحة الوحدة . 10 مترمربع.

(INTEGRATED MODEL OF VALUE ENGINEERING AND RISK MANAGEMENT APPROACHES IN EMPOWERMENT PROJECTS (THE EXTERIOR DESIGN), BEHRANG ASKARI SABZKOHI, Department of Civil Engineering, Faculty of Engineering Islamic Azad University, Central Tehran Branch, Tehran, Iran, Proceedings of 17th IASTEM International Conference, Istanbul Turkey, 26th March 2016.p(1:4) 


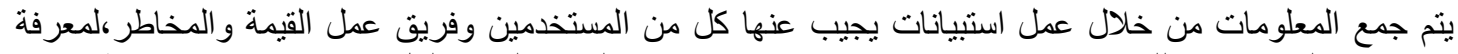

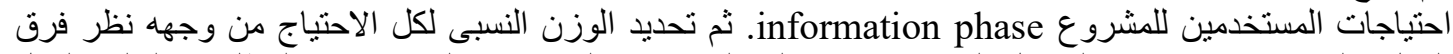

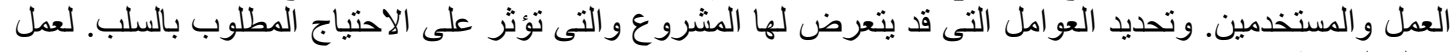

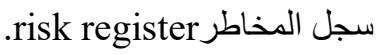

جدول( (1):مستويات احتمالات و وتأثير ات المخاطر .

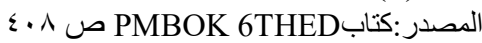

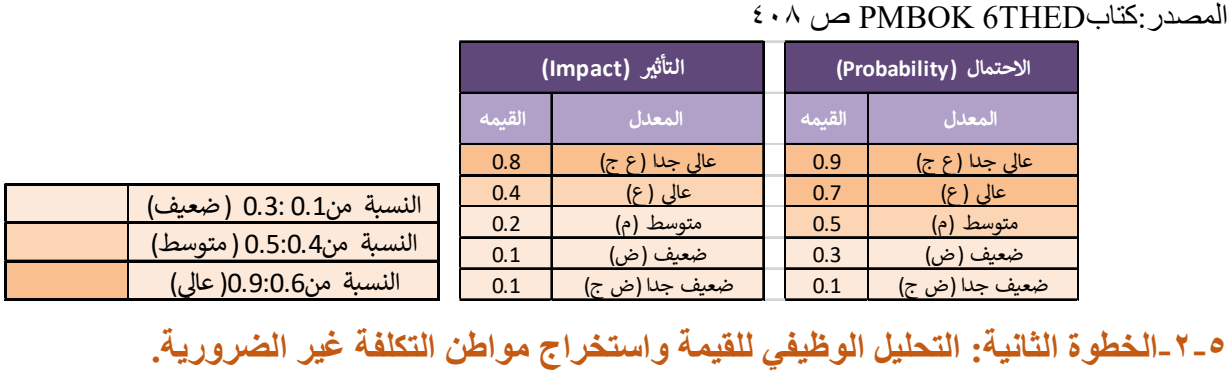

من خلال نتائج تحليل الاستبيانات من نسب للاحتياجات وكذللك عناصر المخاطر و أثرها واحتماليه حدوثها، هناك عدة خطوات Function analysis و هم:

$$
\begin{aligned}
& \text { 1 - تحليل تكلفة المشروع. } \\
& \text { r - تحديد نسبة تأثثير البنود الرئيسية على الاحتياجات. } \\
& \text { r - تحديد نسبة تكلفة احتياجات المستعملين. }
\end{aligned}
$$

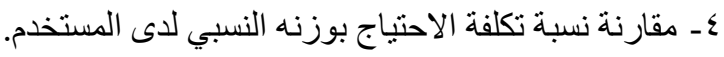

$$
\begin{aligned}
& \text { هـبـ الخطوة الثاثلة: التصليل النوعى والكمى للمخاطر. }
\end{aligned}
$$

يتم تحديد شدة الخطر و هو متوسط حاصل ضرب ابرة احتمال حدوث الخطر فى مدى تأثيره لكل بند.

\begin{tabular}{|c|c|c|c|c|c|c|}
\hline & \multicolumn{5}{|c|}{ المخاطرا (Threats) المخر } & |الاحتمال|ال Probability) \\
\hline \multirow{2}{*}{ نسبه التحليل الككي Quantitative } & 0.72 & 0.36 & 0.18 & 0.09 & 0.045 & 0.9 \\
\hline & 0.56 & 0.28 & 0.14 & 0.07 & 0.035 & 0.7 \\
\hline \multirow[t]{2}{*}{ RISK RESPONSE الاستجابة لمخاطر } & 0.4 & 0.2 & 0.1 & 0.05 & 0.025 & 0.5 \\
\hline & 0.24 & 0.12 & 0.06 & 0.03 & 0.015 & 0.3 \\
\hline \multirow[t]{2}{*}{ قائمة المراقبة WATCH LST } & 0.08 & 0.04 & 0.02 & 0.01 & 0.005 & 0.1 \\
\hline & 0.8 & 0.4 & 0.2 & 0.1 & 0.05 & التاثئر (Impact) \\
\hline
\end{tabular}

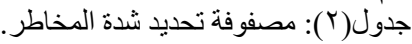

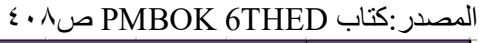

الحالة الأولى(شدة الخطره ه . , • •0 ؛ • , •): فإذا كانت نسبة متوسط شدة الخطر تقع فى منطقة ( قائمة المر اقبة) فيتم

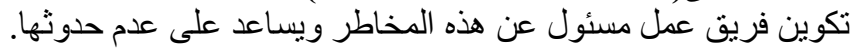

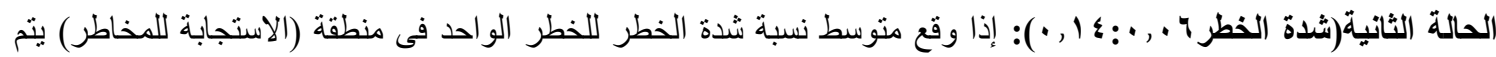
وضع خطة الاستجابة للمخاطر الخطر

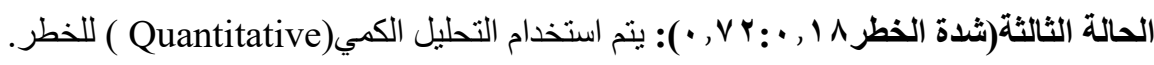
يتم جمع التكلفة الإضافية لبنود عوامل الخطرللحالة الثالثة ليتم إضافتها إلى إجمالى تكلفة المشروع لتغطية عدم حدوث

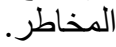

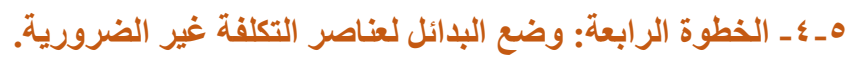


تكامل إدارة القيمة وتحليل المخاطر بمشروعات التثييد والبناء.

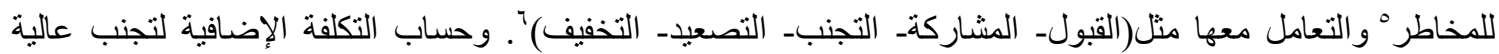

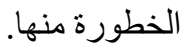

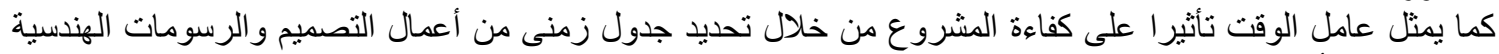

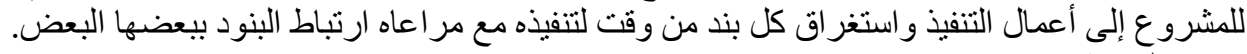

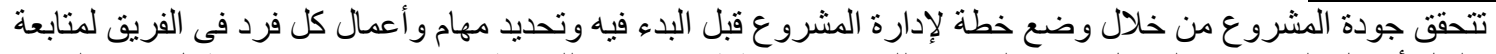

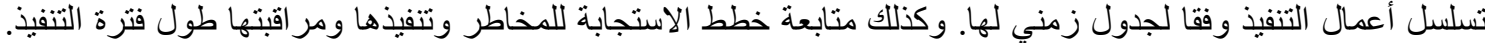

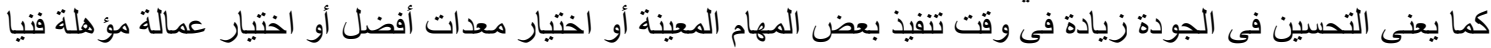

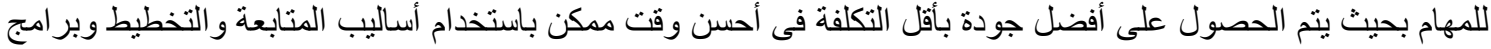

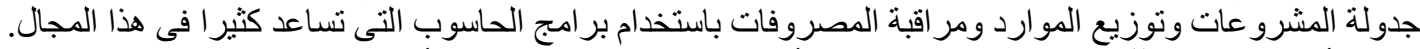

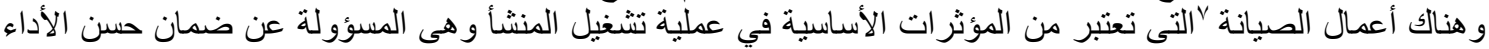

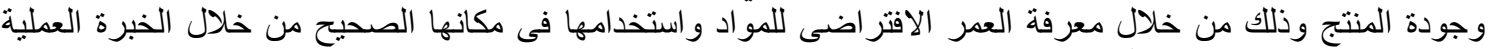

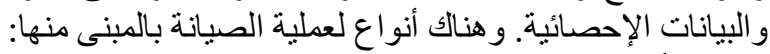

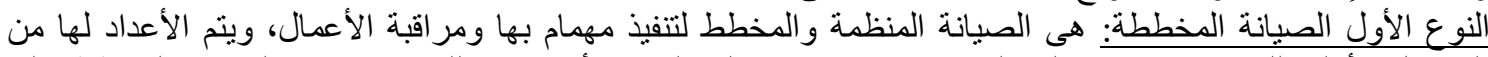

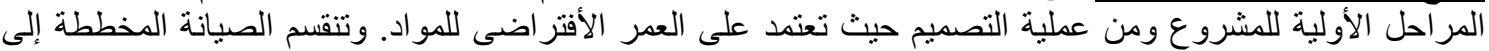

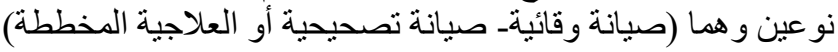

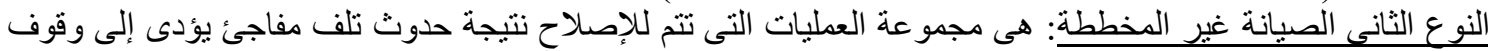

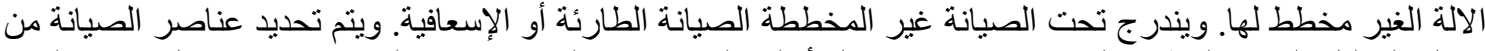

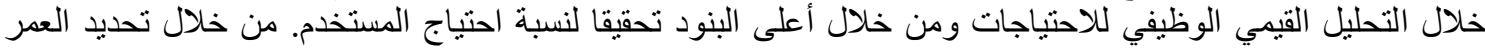

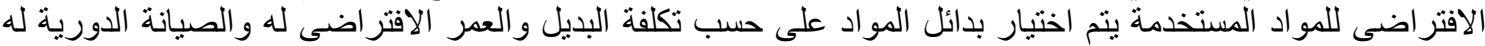

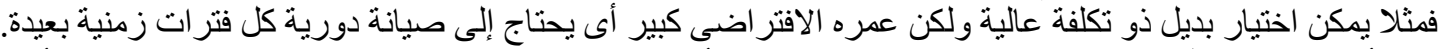

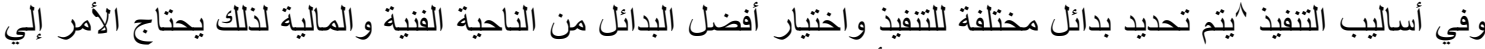

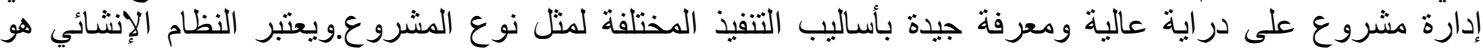

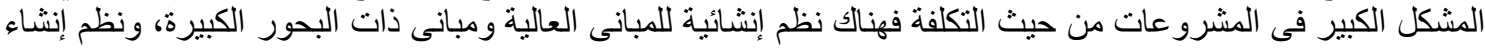

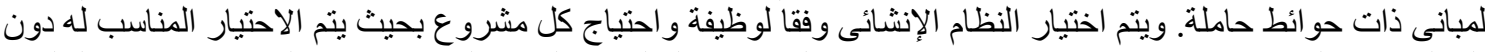

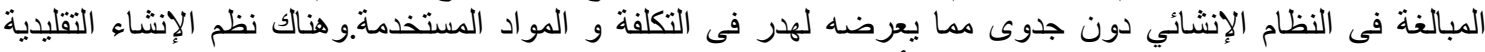

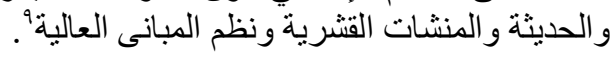

\section{هـ منهجية التكامل بين الهندسة القيمية و إدارة المخاطر فى مشروعات البناء والتشييد:-}

تم استتباط منهج التكامل المفترح الذى يعمل على إثبات أن العامل الرئيس في نجاح تطبيق منهجية التكامل بين الهندسة

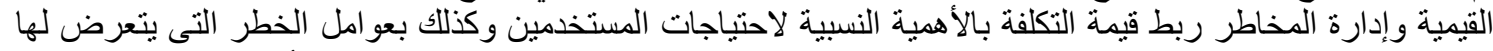

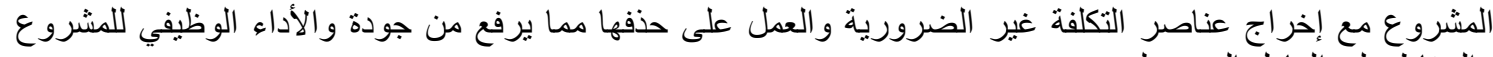
و الحفاظ على العامل الزمنى لـه.

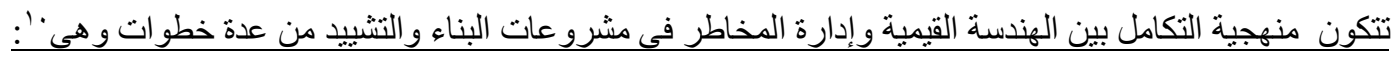
الخطوة الأولى: جمع المعلومات بين الفريقين الهندسة القيمية وإدارة المخاطر.

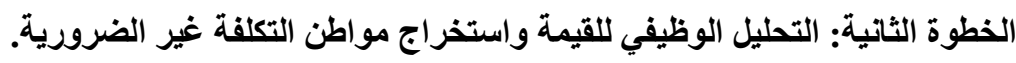
الخطوة الثالثة: التحليل النوعى والكمى للمخاطر.

) A Guide to the Project Management Body of Knowledge, Project Management Institute, Pennsylvania,USA. IVPMBOK, $\left(20^{\circ}\right.$ Project Management Istitute (Guide to the project Management Body of Knowledge )(Newtown square . Pennsylvania,USA ${ }^{\top}$ 2017 6thEd) 437:447).

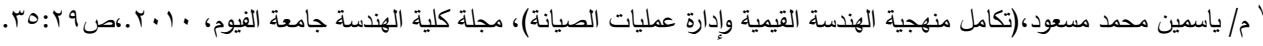

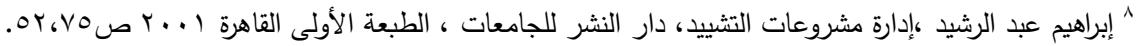

Tall Buildings Structural Systems and Aerodynamic Form Mehmet Halis Günel and Hüseyin Emre Ilgin. • 1 د/ محمد سعيد مصيلحي ، الهندسة القيمية نحو منهج توافقي قيمي للمشروعات الإسكان الحكومي بمصر من خلال التحليل الوظيفي، رسالة 
تكامل إدارة القيمة وتحليل المخاطر بمشروعات التشييد والبناء.

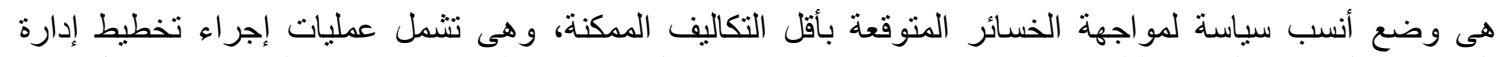

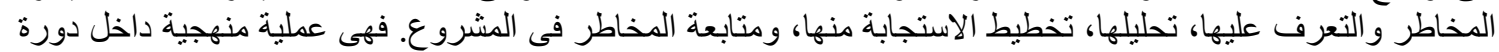

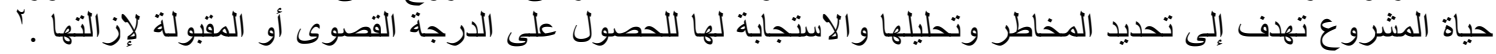

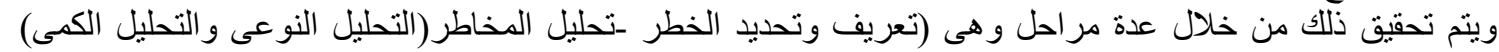

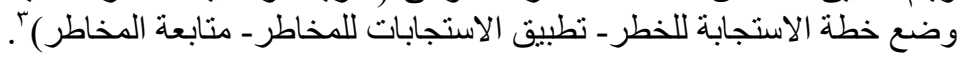

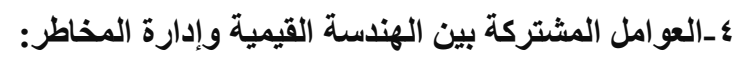

هنالك عوامل مشتركة بين كل من المجالين الهندسة القيمية وإدارة المخاطر والتى تحدد أهداف كلا من المجالين وهم) الجودة).

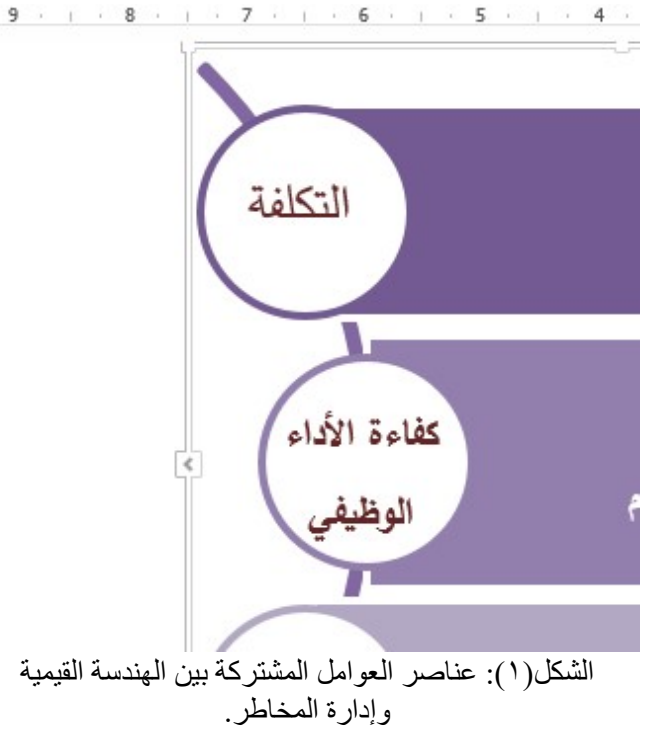
التكلفة- كفاءة الاداء الوظيفيو إدارة المخاطر.

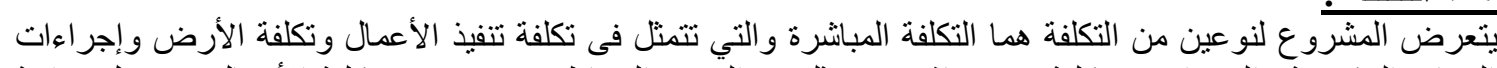

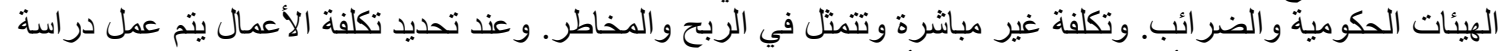

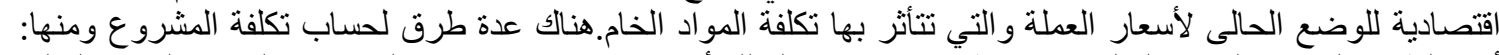

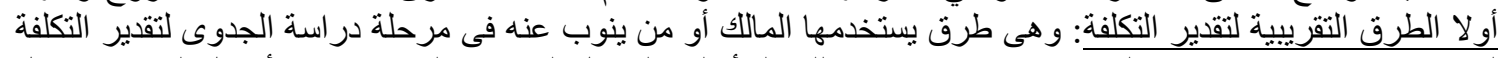

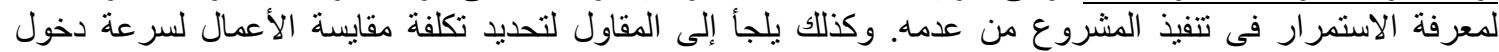

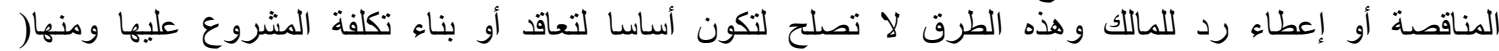

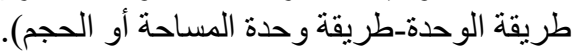

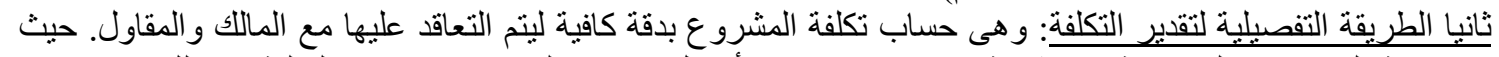

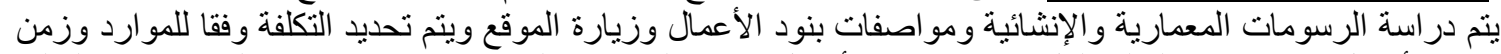

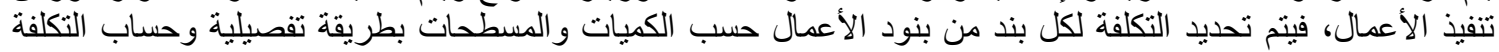

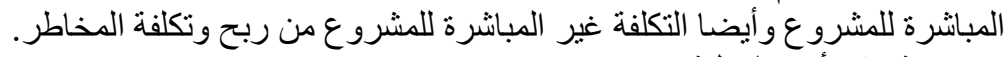

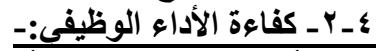

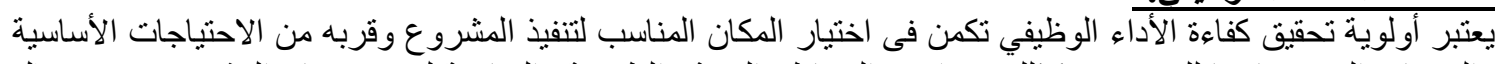

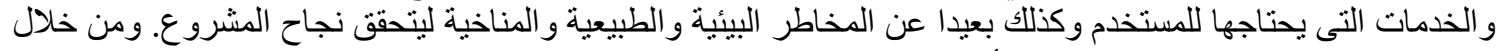

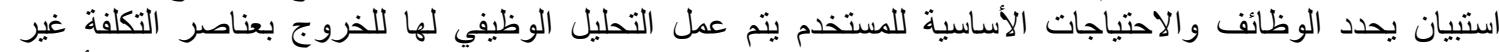

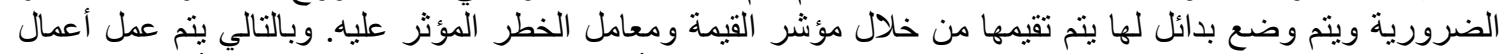

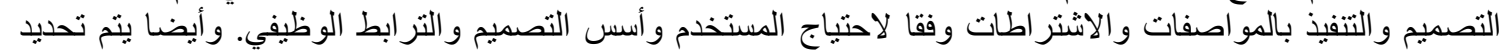
المخاطر التى يتعرض لها المشروع و فقا لاستبيان يجيب عنه فريق المخاطر و المعنيين بالمشروع، لوضئ لوضع خطط الاستجابة

${ }^{2}$ ( Al-Bahar, J and Crandall, K. (1990). "Systematic risk management approach for construction projects". Journal of Construction Engineering and Management, Vol.116, No.3

Project Management Istitute (Guide to the project Management Body of Knowledge )(Newtown square . Pennsylvania,USA ${ }^{r}$ 2017 6thEd)p403:410 ؛ إبراهيم عبد الرشيد ،إدارة مشروعات التشييد، دار النشر للجامعات ، الطبعة الأولى القاهرة ا...... 


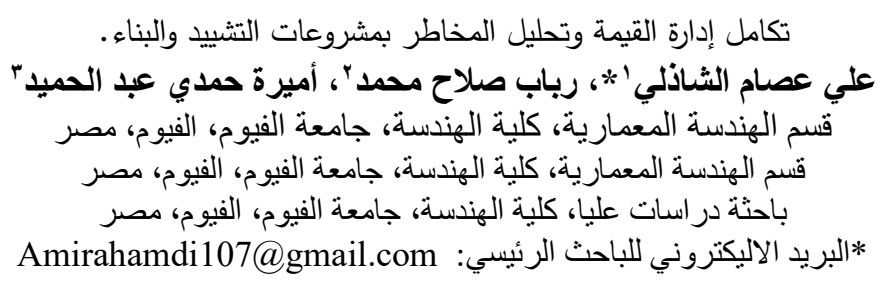

الملخص

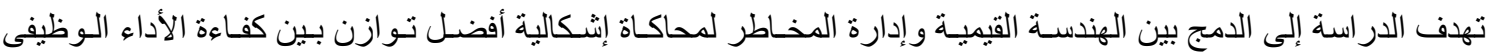

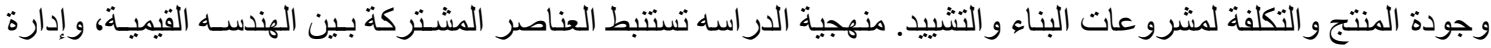

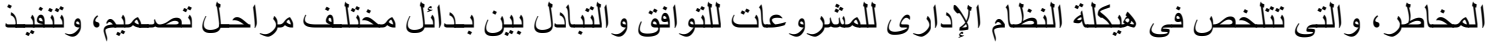

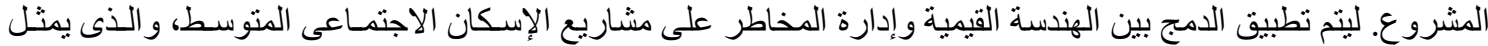

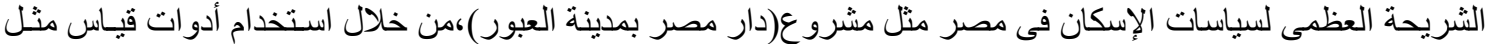

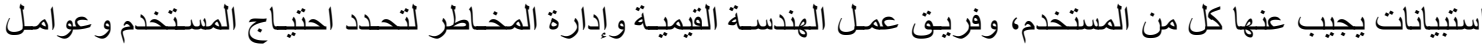

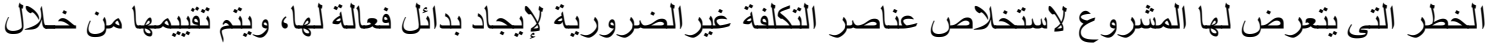

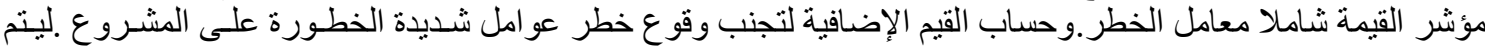

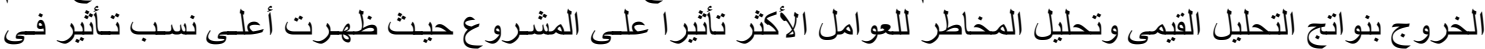

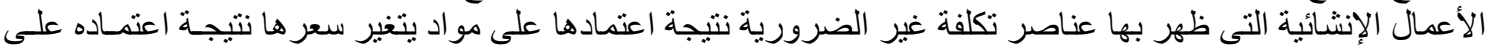

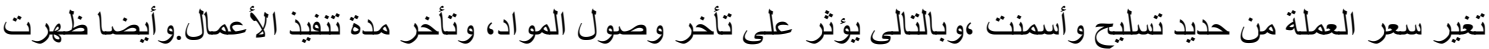

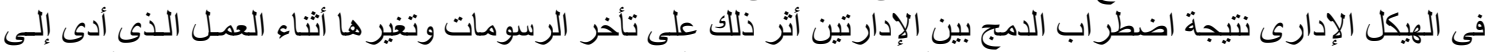

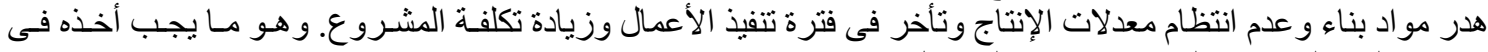
الاعتبار لهذا النوع من المشروعات في المستقبل.

الكلمات الدالة: الهندسة القيمية ـ التكلفة غير الضرورية_ـ الأداء الوظيفي- مؤشر القيمة ــ إدارة المخاطر - التقييم النوعي - التقييم الكمي - معامل الخطر - شدة الخطر.

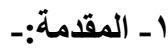

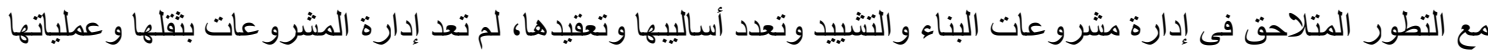

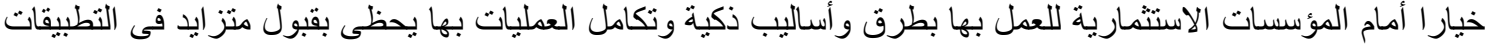

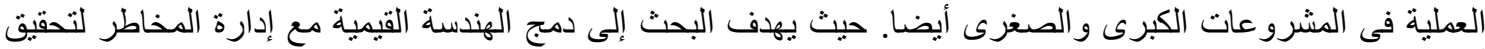

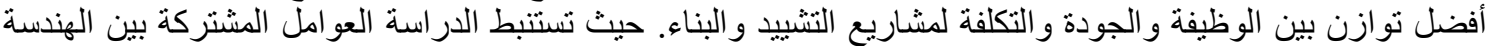

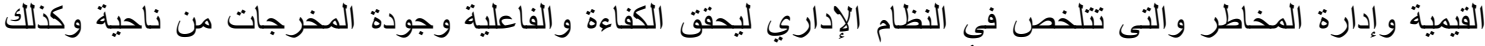

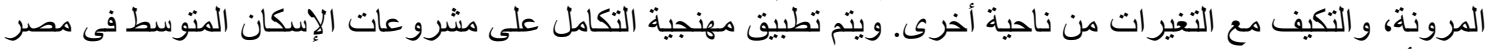

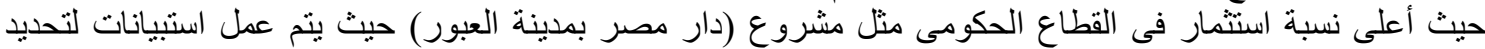

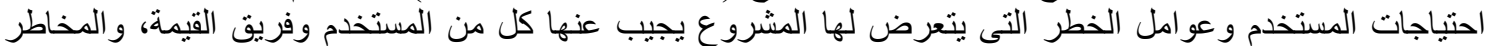

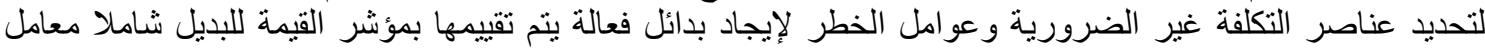

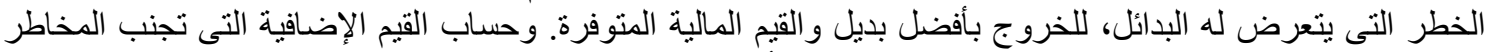

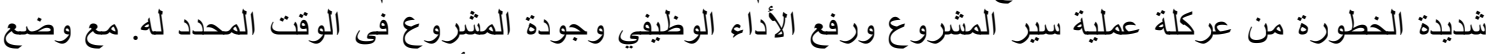

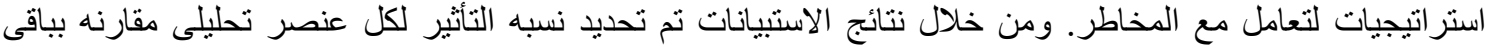
العناصر على مستوى حاله الدراسة. وتتمنل حزمة العناصر من وجهه نظر الهندسة القيمية و إدارة المخاطر للمشروع.

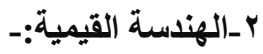

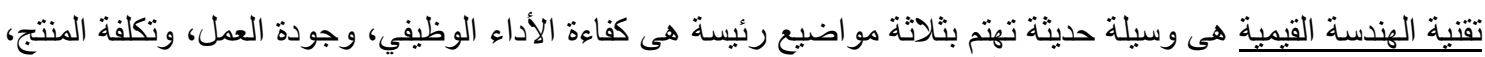

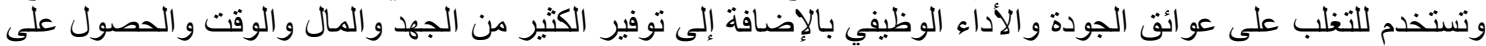

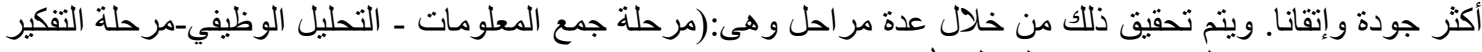

الابتكارى و الإبداعى_التقييم واختيار البدائل) '.

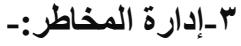

H.M.Harris Jr, Creating value in engineering and construction frim , FMI corporation , New York , 2015 


\title{
INTEGRATION OF MANAGEMENT AND RISK ANALYSIS IN THE CONSTRUCTION
}

\author{
Ali EL Shazly ${ }^{1, *}$, Rabab Salah Mohamed ${ }^{2}$, Amira Hamdi Abd El-Hamed. ${ }^{3}$ \\ ${ }^{1}$ Professor of Architecture Faculty of Engineering - Fayoum University \\ ${ }^{2}$ Lecturer of Architecture Faculty of Engineering - Fayoum University \\ ${ }^{3}$ Graduate Researcher, Faculty of Engineering, University of Fayoum, Fayoum. \\ *Corresponding Author E-mail: Amirahamdi107@gmail.com
}

Received: 20 March $2021 \quad$ Accepted: 27 May 2021

\begin{abstract}
The survey aims to incorporate value engineering and risk management to simulate the issue of striking the optimal balance between the competence of job performance, product quality and building and construction projects cost. The survey methodology extrapolates the common components between value engineering and risk management, which is summarized in the structuring of projects administrative system for compatibility and exchange between the various phases alternatives of project design and implementation. The corporation of value engineering and risk management shall be applied to middle-income housing projects, which represents the largest segment of housing policies in Egypt, such as the project (Dar Misr in Obour City), through the use of measurement tools such as questionnaires answered by both the user, the value engineering and risk management team to determine the user's need and the risk factors that the project is exposed to, to extract the unnecessary costs to find effective alternatives to them, and they are assessed through the value indicator including the risk factor and the calculation of added values to prevent the risk of high-risk factors on the project. In order to produce the results of value and risk analysis of the elements that have the greatest impact on the project, where the highest proportions of impact arose in the construction works in which unnecessary costs arose as a result of their dependence on materials whose price changes according to currency change such as steel and cement, and thus leads to delayed arrival of materials and the delay in works implementation. And also arose in the administrative structure as a result of the disturbance of incorporating between the two departments, the impact of this on the delay of drawings and their change during the work, which led to construction materials wasting, irregularity of production rates, delay in the works implementation period and the increase in the project cost. This shall be taken into account for such types of projects forthcoming.

Keywords: value engineering - unnecessary cost - job performance - value index - risk management - qualitative assessment - quantitative assessment - risk factor - severity of risk.
\end{abstract}

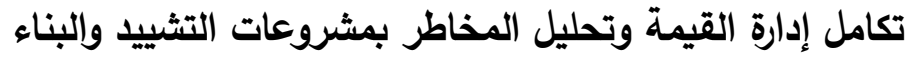

